\title{
Local volatility changes in the Black-Scholes model*
}

\author{
Hans-Peter Bermin ${ }^{\dagger}$ \\ and \\ Arturo Kohatsu-Higa ${ }^{\ddagger}$
}

September 1999

Key words: Contingent claims, hedging, local Vega index, Malliavin calculus, stochastic flows.

\section{JEL classification: G13.}

AMS 1991 subject classification: 60H07, $90 \mathrm{~A} 99$.

Date of entry in Economics Working Paper Series and published in Web page:

\footnotetext{
${ }^{a}$ This paper was prepared while the first author was visiting the Department of Economics at Universitat Pompeu Fabra, Barcelona. The first author is very grateful for the Department's hospitality, and for financial support by the Wallander foundation. The second author was partially supported by a DGES grant.

${ }^{b}$ Lund University, Department of Economics, P.O. Box 7082, 22007 Lund - SWEDEN. e-mail: hans-peter.bermin@nek.lu.se.

${ }^{c}$ Universitat Pompeu Fabra, Department of Economics, Ramon Trias Fargas 25-27, 08005 Barcelona - SPAIN. e-mail: kohatsu@upf.es.
} 


\begin{abstract}
In this paper we address a problem arising in risk management; namely the study of price variations of different contingent claims in the Black-Scholes model due to anticipating future events. The method we propose to use is an extension of the classical Vega index, i.e. the price derivative with respect to the constant volatility, in the sense that we perturb the volatility in different directions. This directional derivative, which we denote the local Vega index, will serve as the main object in the paper and one of the purposes is to relate it to the classical Vega index. We show that for all contingent claims studied in this paper the local Vega index can be expressed as a weighted average of the perturbation in volatility. In the particular case where the interest rate and the volatility are constant and the perturbation is deterministic, the local Vega index is an average of this perturbation multiplied by the classical Vega index. We also study the well-known goal problem of maximizing the probability of a perfect hedge and show that the speed of convergence is in fact dependent of the local Vega index.
\end{abstract}




\section{Introduction}

In this paper we will analyze a sensitivity problem with respect to variations in parameters arising in risk management. However, to keep things simple we will place ourselves in the wellknown Black-Scholes model. In order to fix our terminology we consider a finite time interval $\left[0, T^{*}\right]$ and assume that the basic market consists of two assets: one locally risk free asset of price $B(\cdot)$ and one stock of price $S(\cdot)$. The interpretation of the locally risk free asset is as usual that of a bank account where money grows at the short interest rate $r$. The asset prices are modelled by the (stochastic) differential equations

$$
\begin{aligned}
& \left\{\begin{array}{l}
d B(t)=r B(t) d t \\
B(0)=1
\end{array}\right. \\
& \left\{\begin{array}{l}
d S(t)=\alpha S(t) d t+\sigma S(t) d W(t) \\
S(0)=S_{0}
\end{array}\right.
\end{aligned}
$$

where we assume that the coefficients $r, \alpha, S_{0}$ and $\sigma$ are strictly positive constants. Here we let $\left\{W(t): 0 \leq t \leq T^{*}\right\}$ denote a Brownian motion defined on the complete probability space $(\Omega, \mathcal{F}, P)$ and we let $\mathbb{F}=\left\{\mathcal{F}_{t}: 0 \leq t \leq T^{*}\right\}$ denote the natural filtration generated by the $\sigma$ fields $(W(s): 0 \leq s \leq t)$ and completed by the $P$-null sets of $\mathcal{F}$. It is well known that the basic market is free of arbitrage opportunities and complete, see e.g. the classical article by Harrison and Pliska (1981). Hence, for any sufficiently integrable $\mathcal{F}_{T}$-measurable contingent claim $G$, we can define the corresponding price process $\Pi(\cdot)$ according to:

$$
\Pi(t)=B(t) B(T)^{-1} E_{Q}\left[G \mid \mathcal{F}_{t}\right],
$$

where $Q$ is the unique equivalent martingale measure under which the process $V(\cdot):=W(\cdot)-$ $\int_{0}^{\cdot}\left(\frac{r-\alpha}{\sigma}\right) d s$ is a Brownian motion. Consequently, prices are to be computed given the dynamics

$$
\left\{\begin{array}{l}
d S(t)=r S(t) d t+\sigma S(t) d V(t) \\
S(0)=S_{0}
\end{array}\right.
$$

of the stock. For technical convenience, we will assume from now on that the contingent claims to be studied are square integrable, i.e. belong to the space $L^{2}(\Omega, \mathcal{F}, Q)$. Note also that the maturity of a contingent claim $T$ is an arbitrary value in the interval $\left[0, T^{*}\right]$.

Of course, the Black-Scholes model is a simplified model of reality. This is seen, for example, by taking the prices of traded options as well as the interest rate as given and thereafter solving backwards for the implied volatility, see e.g. Dupire (1994) for further details. As a result one normally finds that the implied volatilities are not constant over time, which contradicts the specification of the stock price in (1.4). However, taking into account the simplicity of the Black-Scholes model it is an outstanding benchmark. The only parameter to be estimated is the volatility $\sigma$, and thereafter closed form solutions for most contingent claims can quite easily be 
obtained. Hence, the derived contingent claim prices will depend upon the estimated volatility, and therefore it is of course of great interest to know how sensitive the derived prices are to mis-specifications of $\sigma$. Clearly, the natural way to carry out this sensitivity analysis is to study the derivative of the contingent claim prices with respect to the volatility, i.e. $\frac{\partial \Pi}{\partial \sigma}(\cdot)$ which in the financial field is known as the Vega index of the contingent claim. However, as we will show in this paper, there are other ways as well. To see this, consider a generalized version of the Black-Scholes model where the dynamics of the stock price are given by:

$$
d S(t)=r(t) S(t) d t+\sigma(t) S(t) d V(t)
$$

where $r(\cdot)$ and $\sigma(\cdot)$ are strictly positive deterministic functions. The advantage of the generalized version is of course that we now can, more or less, calibrate the volatility structure to the implied volatilities obtained from the prices of the traded options at the market. However, this procedure gives us a set of implied volatilities for different points in time, to which we have to fit the continuous volatility function $\sigma(\cdot)$, hence again we are interested in some kind of sensitivity analysis. This time, though, things are not as obvious as before since we now would like to take the derivative of the contingent claim prices with respect to the deterministic volatility function. Clearly, this cannot be done so instead we have to consider directional derivatives. However, depending upon in which direction we perturb the volatility function, we get different directional derivatives. Although the increasing complexity makes it harder to use the result for classical sensitivity analysis, it gives us the possibility to address very specific questions like: what happens to the contingent claim prices if the next two weeks will be a very unstable period, or what happens to the contingent claim prices if the volatility will drop in a month as predicted by a time series analysis. Of course, such questions are of great interest and arises naturally in financial risk management. Hence, the conclusion to be drawn is that the commonly used Vega index is not the only candidate for studying price variations due to changes in volatility.

The quantity we will propose to study is therefore the local Vega index:

$$
\left.\frac{\partial \Pi^{\varepsilon}}{\partial \varepsilon}(0)\right|_{\varepsilon=0}=\left.\frac{\partial}{\partial \varepsilon} B(T)^{-1} E_{Q}\left[G^{\varepsilon}\right]\right|_{\varepsilon=0},
$$

where now $B(\cdot):=\exp \left(\int_{0}^{\cdot} r(s) d s\right)$. The contingent claim $G^{\varepsilon}$ has the same form as $G$ except that the underlying security $S(\cdot)$ is replaced by the perturbed stock price $S^{\varepsilon}(\cdot)$ defined as the solution to the stochastic differential equation:

$$
\left\{\begin{array}{l}
d S^{\varepsilon}(t)=r(t) S^{\varepsilon}(t) d t+\sigma_{\varepsilon}\left(t, S^{\varepsilon}(t)\right) d V(t) \\
S^{\varepsilon}(0)=S_{0}
\end{array}\right.
$$

where $\sigma_{\varepsilon}(\cdot, \cdot)$ is a deterministic function such that the above equation has a unique a.s. strictly positive pathwise solution. For technical convenience we assume throughout this paper that $\varepsilon$ belongs to the compact set $[0, \bar{\varepsilon}]$ for some $\bar{\varepsilon} \geq 1$, and that the following assumption, which we will refer to as assumption $\mathrm{A} 1$, is satisfied. 
Assumption (A1) The volatility $\sigma_{\varepsilon}(t, x):=\sigma(t) x+\varepsilon \hat{\sigma}(t, x) \geq 0$, satisfies $0<\sigma_{\min } \leq \sigma(t) \leq$ $\sigma_{\max }$ for all $t \in\left[0, T^{*}\right], x \geq 0$, and $\varepsilon \leq \bar{\varepsilon}$. Furthermore, we assume that $\hat{\sigma}(t, x)$ is infinitely differentiable in $x$ with bounded partial derivatives of any order uniformly in $t$, and that $\sigma_{\varepsilon}(t, x)$ is bounded away from zero in a neighborhood of $\left(0, S_{0}\right)$ uniformly in $\varepsilon$. The short interest rate $r(\cdot)$ and $\hat{\sigma}(\cdot, 0)$ are bounded functions.

Note that under A1 we allow the perturbations to be random. From a practical point of view this may be interesting since it gives us the possibility to study different scenarios. For instance we could model an increase in anticipated volatility when the stock price reaches some threshold level. Hence, assumption A1 is indeed very general, and in fact for some of the contingent claims that are to be studied it is too general. We will therefore, sometimes, use the following stronger conditions, which we will refer to as assumption A2.

Assumption (A2) $r(t)=r, \sigma(t)=\sigma$, and $\hat{\sigma}(t, x)=\hat{\sigma}(t) x$ for all $t \in\left[0, T^{*}\right]$ and $x \geq 0$. The deterministic measurable function $\sigma+\varepsilon \hat{\sigma}(\cdot)$ is strictly positive and bounded for all $\varepsilon$. More precisely we assume without loss of generality that $0<\sigma_{\min } \leq \sigma+\varepsilon \hat{\sigma}(t) \leq \sigma_{\max }$ for all $\varepsilon, t$.

Note that under A2, we can intuitively relate the local Vega index to the usual Vega index since the stock price $S(\cdot)$ according to (1.4) then corresponds to the case where $\varepsilon=0$ in (1.6). For the sake of simplicity we will in this case denote $S^{0}(\cdot)$ simply by $S(\cdot)$, and we will use this kind of convention for any quantity that depends on $\varepsilon$ when we are working under A2. However, note that whenever we consider the operator $\frac{\partial}{\partial \sigma}$, as in the case of the Vega index, we assume that the stock price is defined by (1.4).

The main question of interest is to study the new concept of local Vega index in (1.5) and compute it when possible. Under A2, we will also study its relationship with the Vega index $\frac{\partial \Pi}{\partial \sigma}(\cdot)$ and give conditions on the deterministic function $\hat{\sigma}(\cdot)$ for the two sensitivity indices to coincide.

To motivate the study we consider a financial manager who is responsible for the trading activity at some company. The manager is aware of the fact that mis-specifications or changes of the volatility can drastically alter the balance of the trading activity. Therefore, the manager may impose the restriction to the traders that together they must be more or less "Vega neutral", i.e. $\sum_{i} \frac{\partial \Pi_{i}}{\partial \sigma}(\cdot) \approx 0$ where the sum is taken over every contingent claim traded by the company. Hence, by following this strategy the manager is on average protected from volatility changes. Now, let us suppose that one day the manager poses the question: what will happen to our balance if the volatility will rise in two months due to a political meeting that will take place just before. To answer such specific questions, we already know that we have to use directional derivatives, hence in this case the manager should use a strategy setting $\left.\sum_{i} \frac{\partial \Pi_{i}^{\epsilon}}{\partial \varepsilon}(\cdot)\right|_{\varepsilon=0} \approx 0$ and set the local volatility function $\hat{\sigma}(\cdot, \cdot)$ according to previous analyses or beliefs. Consequently, it is of course interesting to see if this new strategy will produce other results than the well-known 
Vega neutrality concept. The conclusion we will reach is that in some particular cases these two strategies will give the same conclusions.

The relationship between the two approaches comes out rather straightforward if it is possible to obtain a closed form solution for the expectation $E_{Q}\left[G^{\varepsilon}\right]$, however as we will see in the next sections even if this is not the case we will still be able to compute the local Vega index and relate it to the classical Vega index. Nevertheless, in order to start our analysis let us consider an example where this relationship can be deduced easily.

Example 1.1 Let us consider a standard call option with payoff $G^{\varepsilon}=\max \left(S^{\varepsilon}(T)-K, 0\right)$ for some constant strike price $K$ and assume A2. It is easily verified that at time 0 the price is given by

$$
\Pi^{\varepsilon}(0)=S_{0} N\left(d_{1}^{\varepsilon}\right)-e^{-r T} K N\left(d_{1}^{\varepsilon}-\sqrt{\Sigma^{\varepsilon}}\right),
$$

where $N(\cdot)$ denotes the cumulative distribution function of a standard normal random variable, and $d_{1}^{\varepsilon}$ is defined by

$$
d_{1}^{\varepsilon}=\frac{\ln \left(S_{0} / K\right)+r T+\frac{1}{2} \Sigma^{\varepsilon}}{\sqrt{\Sigma^{\varepsilon}}} \quad ; \quad \Sigma^{\varepsilon}=\int_{0}^{T}(\sigma+\varepsilon \hat{\sigma}(t))^{2} d t .
$$

Straightforward calculations then gives, denoting $\varphi(\cdot)=\frac{d N}{d x}(\cdot)$, that

$$
\left.\frac{\partial \Pi^{\varepsilon}}{\partial \varepsilon}(0)\right|_{\varepsilon=0}=S_{0} \varphi\left(d_{1}^{0}\right) \frac{1}{\sqrt{T}} \int_{0}^{T} \hat{\sigma}(t) d t \quad ; \quad \frac{\partial \Pi^{0}}{\partial \sigma}(0)=S_{0} \varphi\left(d_{1}^{0}\right) \sqrt{T} .
$$

Finally, since $\frac{\partial \Pi}{\partial \sigma}(0)=\frac{\partial \Pi^{0}}{\partial \sigma}(0)$, we get the relationship

$$
\left.\frac{\partial \Pi^{\varepsilon}}{\partial \varepsilon}(0)\right|_{\varepsilon=0}=\frac{1}{T} \int_{0}^{T} \hat{\sigma}(t) d t \frac{\partial \Pi}{\partial \sigma}(0) .
$$

In fact there are a lot of interesting results that are worth to be pointed out in this very simple example. First we see that the two approaches are identical if $\int_{0}^{T} \hat{\sigma}(t) d t=T$ for any maturity $T \in\left[0, T^{*}\right]$. This implies that for standard call options the usual derivative $\frac{\partial \Pi}{\partial \sigma}(\cdot)$ is identical to the directional derivative with an uniform perturbation, i.e. $\hat{\sigma}(\cdot)=1$ a.e. $-\lambda$ where from now on $\lambda$ denotes the Lebesgue measure. In fact, as we will show in the forthcoming sections, this is a result that is true in general and not only for standard options. Moreover, for standard options the manager's personal belief about the future market behavior is irrelevant as long as $\int_{0}^{T} \hat{\sigma}(t) d t=T$. Hence, if the manager anticipates that the volatility will first drop and then rise symmetrically around the uniform level $\hat{\sigma}(\cdot)=1$, the result will be identical to the converse scenario with a initial rise followed by a drop. This result, however, is not true for other type of options as we will see later on. If on the other hand, the manager believes that there will be significant changes in the volatility, the two approaches can give quite different results. 
For all the options studied in this paper, the local Vega index can be expressed as a weighted average of the perturbation in volatility. Under A2, the local Vega index is an average of this perturbation multiplied by the classical Vega index. In particular, we will conclude that for path-dependent options the change in option prices due to a perturbation of volatility decreases in importance as maturity is approached. This is natural since the payoff of a path-dependent option depends on the whole path of the stock price. Hence, a change of volatility at the beginning of the time to maturity will affect almost the whole path of the underlying security, while a change of volatility at the end of the time to maturity will only affect a small part of the path of the stock price. In the case of the lookback option, we find in comparison to example 1.1, that not only the average of change in volatility is an important quantity that determines the change in price, but also the modulus of continuity plays a role in the sensitivity analysis.

The type of results that we exposed here are also quite related with concepts of risk management for portfolios. In order to see this, let us consider the case where we can calculate explicitly $E_{Q}[G]$ but not the perturbed expected value $E_{Q}\left[G^{\varepsilon}\right]$. Under assumption A2 we show, for example, that for all the options studied in this paper a local volatility change of the form $\hat{\sigma}(\cdot) \geq 0$ implies the inequality $\Pi(0) \leq \Pi^{\varepsilon}(0)$, see El Karoui et al. (1998) and Hobson (1997) for related results. Consequently, if we use the lower bound $\Pi(0)$ as the initial amount of money we can never obtain a perfect hedge of the contingent claim $G^{\varepsilon}$. Therefore we study the problem of maximizing a perfect hedge and we show that the local Vega index is an important quantity in determining the speed at which the probability of maximizing a perfect hedge goes to one as $\varepsilon$ goes to zero.

Since, the local Vega index measures the local change in prices due to a perturbation in volatility in a quantitative manner, we can properly call it an index. Hence, a big value for this index corresponds to a big change in the option price. This contrasts with the qualitative results of El Karoui et al. (1998) and Hobson (1997).

To carry out the analysis in a general way we cannot assume that there exists a closed form solution to the expected value $E_{Q}\left[G^{\varepsilon}\right]$. Instead we will use the natural approach of derivation on Wiener space. For this reason we introduce Malliavin calculus and in particular the integration by parts formula in the next section. Malliavin calculus is a natural tool to use in finance as it gives information about hedging portfolios through the concept of stochastic derivation, see e.g. Bermin (1998a), (1998b), and Karatzas and Ocone (1991). Recently, Malliavin calculus has been applied in other areas through the integration by parts formula which allows for analysis of non-smooth functions of smooth random variables, see e.g. Fournié et al. (1997) who used this technique to study the numerical simulation of greeks.

It is a natural extension to obtain the formulas that we give in the next sections in a general framework of underlying stock prices modelled by multi dimensional diffusion processes that satisfy some type of Hörmander condition. As our interest, though, is to show that the concept of sensitivity can be developed and computed explicitly we have decided to leave the extensions 
to another publication. The techniques to study higher order derivatives of option prices are of course similar to the ones we use throughout the paper.

We start in Section 2 with some basic properties of Malliavin Calculus and stochastic flows. In Section 3.1 we consider so called simple options, i.e. European type options based on the terminal value of the underlying security. In Section 3.2 we study the case of Asian type options in order to show that the technique used in Section 3.1 can be extended to any path-dependent option as long as the smoothness of the random variables involved is preserved. In Section 3.3 we deal with a case where the random variables are not smooth. In particular we consider lookback options that depend on the supremum of the path. We believe that the analysis for other pathdependent options that are dependent on max-min values should be similar. In Section 4 we try to find a further interpretation of the local Vega index. We investigate the probability of perfect hedging in the case where a perfect hedge cannot be achieved. We prove that the rate of convergence of such a probability, as the information on the volatility becomes revealed, is also determined by the local Vega index. Finally we close with some conclusions and two Appendices.

\section{Stochastic flows and Malliavin calculus}

In this section we give a brief account of some elementary properties of stochastic flows and Malliavin calculus. For far more general results see for instance Protter (1990) and Nualart (1995) respectively. It is well-known that the unperturbed stock price $S^{0}(\cdot)$, according to (1.6), admits the unique solution

$$
S^{0}(t)=S_{0} \exp \left(\int_{0}^{t}\left(r(s)-\frac{1}{2} \sigma(s)^{2}\right) d s+\int_{0}^{t} \sigma(s) d V(s)\right) \quad ; \quad 0 \leq t \leq T^{*} .
$$

Since $S^{0}(\cdot)$ coincide with $S(\cdot)$ under A2, we see that $S(t)=S_{0} \exp \left(\left(r-\frac{1}{2} \sigma^{2}\right) t+\sigma V(t)\right)$ and consequently we can without ambiguity, for all $t \in\left[0, T^{*}\right]$, define the derivative processes:

$$
\frac{d S}{d S_{0}}(t)=S(t) / S_{0} \text { and } \frac{d S}{d \sigma}(t)=-[\sigma t-V(t)] S(t)
$$

In order to define the derivative process of $S^{\varepsilon}(\cdot)$ with respect to $\varepsilon$, we first use theorem 39 in Protter (1990) to ensure that we can always choose versions of $\left\{S^{\varepsilon}(t): 0 \leq t \leq T^{*}\right\}$ which are continuously differentiable with respect to $\varepsilon$ for each $(t, \omega) \in\left[0, T^{*}\right] \times \Omega$. Now, we let the stochastic process $\left\{Z^{\varepsilon}(t): 0 \leq t \leq T^{*}\right\}$ denote the derivative process of $S^{\varepsilon}(\cdot)$ with respect to $\varepsilon$, defined as the solution to the stochastic differential equation:

$$
\left\{\begin{array}{l}
d Z^{\varepsilon}(t)=r(t) Z^{\varepsilon}(t) d t+\sigma_{\varepsilon}^{\prime}\left(t, S^{\varepsilon}(t)\right) Z^{\varepsilon}(t) d V(t)+\hat{\sigma}\left(t, S^{\varepsilon}(t)\right) d V(t) \\
Z^{\varepsilon}(0)=0
\end{array}\right.
$$

where $\sigma_{\varepsilon}^{\prime}$ denotes the derivative with respect to the space variable. 
Lemma 2.1 The solution to the stochastic differential equation (2.2) is given by

$$
Z^{\varepsilon}(t)=-\left(\int_{0}^{t} \mathcal{E}^{\varepsilon}(s)^{-1} \sigma_{\varepsilon}^{\prime}\left(s, S^{\varepsilon}(t)\right) \hat{\sigma}\left(s, S^{\varepsilon}(s)\right) d s-\int_{0}^{t} \mathcal{E}^{\varepsilon}(s)^{-1} \hat{\sigma}\left(s, S^{\varepsilon}(s)\right) d V(s)\right) \mathcal{E}^{\varepsilon}(t)
$$

where $\mathcal{E}^{\varepsilon}(t):=\exp \left(\int_{0}^{t}\left(r(s)-\frac{1}{2} \sigma_{\varepsilon}^{\prime}\left(s, S^{\varepsilon}(s)\right)^{2}\right) d s+\int_{0}^{t} \sigma_{\varepsilon}^{\prime}\left(s, S^{\varepsilon}(s)\right) d V(s)\right)$. Note especially that

$$
\mathcal{E}^{0}(t)=\exp \left(\int_{0}^{t}\left(r(s)-\frac{1}{2} \sigma(s)^{2}\right) d s+\int_{0}^{t} \sigma(s) d V(s)\right)=: S^{0}(t) / S_{0},
$$

and in particular under A2:

$$
Z^{0}(t)=-\left(\sigma \int_{0}^{t} \hat{\sigma}(s) d s-\int_{0}^{t} \hat{\sigma}(s) d V(s)\right) S(t)
$$

Proof. The solution is constructed using theorem 52 in Protter (1990). However, the proof can also be obtained directly from the Ito formula.

For the sake of simplicity we will from now on use the short hand notation $Z(\cdot)$ for $Z^{0}(\cdot)$ just as for the stock prices whenever we are working under A2.

Remark 2.1 If we assume A2, and combine lemma 2.1 with (2.1), we see that a.s. for all $t \in\left[0, T^{*}\right], \frac{d S}{d \sigma}(t)=Z(t)$ if and only if $\left(\sigma \int_{0}^{t} \hat{\sigma}(s) d s-\int_{0}^{t} \hat{\sigma}(s) d V(s)\right)=(\sigma t-V(t))$. This will of course be the case only when $\hat{\sigma}(\cdot)=1$. Hence, we recover the same result as we found in the introductory example for standard options.

Now we introduce some concepts of Malliavin calculus. Note that under assumption A2, we can work directly on the probability space $(\Omega, \mathcal{F}, Q)$ since the Brownian motions $W(\cdot)$ and $V(\cdot)$ generate the same filtration, see Karatzas and Ocone (1991) for details. In general though, this does not have to be the case. However, since we will only deal with quantities in expectation and all our stochastic differential equations have strong solutions, we can always change the underlying probability space accordingly so that the concepts of Malliavin Calculus can be applied there. We will do this if necessary without further mentioning.

We will use the following version of the integration by parts formula. Let the stochastic variable $F \in \mathbb{D}^{1,2}$ (for definitions, see Nualart (1995)) and the stochastic process $u(\cdot) \in \operatorname{dom}(\delta)$, then

$$
E_{Q}[F \delta(u)]=E_{Q}\left[\int_{0}^{T^{*}} u(t) D_{t} F d t\right] .
$$

Here $\delta(u)$ denotes the Skorohod integral of $u(\cdot)$ which coincides with the usual stochastic integral if $u(\cdot)$ is adapted, while $D$. denotes the Malliavin derivative defined in $\mathbb{D}^{1,2}$. We remind the reader that $D_{t} \int_{0}^{T^{*}} g(s) d V(s)=g(t)$ for deterministic functions $g(\cdot) \in L^{2}\left(\left[0, T^{*}\right]\right)$, and that 
the usual chain rule applies in the sense that if $\psi(\cdot)$ is a deterministic Lipschitz function and $F$ is a stochastic variable in $\mathbb{D}^{1,2}$, having a density, then $\psi(F) \in \mathbb{D}^{1,2}$ with

$$
D_{t} \psi(F)=\frac{d \psi}{d x}(F) D_{t} F \text {. }
$$

In particular, it follows that $D_{t} S^{0}(s)=\sigma(t) S^{0}(s) \mathbf{1}_{t \leq s}$. Finally, we state the Clark-Ocone formula which says that any $\mathcal{F}_{T^{-}}$-measurable stochastic variable $F \in \mathbb{D}^{1,2}$ has the representation

$$
F=E_{Q}[F]+\int_{0}^{T} E_{Q}\left[D_{t} F \mid \mathcal{F}_{t}\right] d V(t) .
$$

For more details about these concepts we refer the reader to Nualart (1995).

Remark 2.2 The space $\mathbb{D}^{1,2}$ is a dense subspace of $L^{2}(\Omega, \mathcal{F}, Q)$ and therefore it is possible to extend the Clark-Ocone formula. However, for $F \in L^{2}(\Omega, \mathcal{F}, Q)$ we generally have to consider D.F in the distributional sense, see e.g. Üstünel (1997) and Bermin (1998a).

\section{The effect of local volatility changes}

In this section we will start by proving a slight generalization of Example 1 using the tools of derivation of stochastic flows and the integration by parts formula of Malliavin calculus. In particular, we analyze the change in prices due to anticipated volatility changes for different kinds of European contingent claims. The purpose is to compute the local Vega index $\left.\frac{\partial \Pi^{\varepsilon}}{\partial \varepsilon}(0)\right|_{\varepsilon=0}$, and under assumption A2 derive relations between the local Vega index and the classical Vega index $\frac{\partial \Pi}{\partial \sigma}(0)$. It will be shown that these relations depend very much on the specific contingent claims that are treated. In order to define different kinds of contingent claims we need the concept of a payoff function and here we follow El Karoui et al. (1998).

Definition 3.1 A payoff function is a convex function $\Phi(\cdot)$, defined on $(0, \infty)$ and having bounded one-sided derivatives, that is

$$
\left|\Phi^{\prime}(x \pm)\right| \leq C \quad ; \quad \forall x
$$

for a positive constant $C$.

We recall that for any convex function $\Phi(\cdot): \mathbb{R}_{+} \rightarrow \mathbb{R}$, there is a countable set $N \subset \mathbb{R}_{+}$such that $\Phi(\cdot)$ is differentiable on $\mathbb{R}_{+} \backslash N$ and

$$
\Phi^{\prime}(x)=D^{+} \Phi(x)=D^{-} \Phi(x) \quad ; \quad x \in \mathbb{R}_{+} \backslash N,
$$

where $D^{+} \Phi(\cdot)$ (respectively, $D^{-} \Phi(\cdot)$ ) denotes the derivative of $\Phi(\cdot)$ taken from the right (respectively, from the left). The second derivative $\Phi^{\prime \prime}(\cdot)$ may not exist so we define the second derivative measure $\varsigma(\cdot)$ on $(\mathbb{R}, \mathcal{B}(\mathbb{R}))$ by

$$
\varsigma([a, b))=D^{-} \Phi(b)-D^{-} \Phi(a) \quad ; \quad-\infty<a<b<+\infty,
$$


such that $\varsigma(\cdot)$ is positive and $\varsigma(d x)=\Phi^{\prime \prime}(x) d x$ if $\Phi^{\prime \prime}(\cdot)$ exists. In general, $\varsigma(d x)=\Phi^{\prime \prime}(x) d x+$ $m(d x)$, where $m(\cdot)$ is a positive measure which is singular with respect to the Lebesgue measure and $\Phi^{\prime \prime}(\cdot)$ is defined as the second derivative of $\Phi(\cdot)$ whenever it exists and zero otherwise. From now on, though, we will use the second derivative in a formal sense and interpret expressions like $E_{Q}\left[\Phi^{\prime \prime}(F)\right]$ with $F$ being a random variable having a smooth density $f_{F}(\cdot)$, by

$$
E_{Q}\left[\Phi^{\prime \prime}(F)\right]:=\int_{0}^{\infty} f_{F}(x) \varsigma(d x)=\int_{0}^{\infty} f_{F}(x) \Phi^{\prime \prime}(x) d x+\int_{0}^{\infty} f_{F}(x) m(d x) .
$$

In general, our assertions do hold under greater generality for the behavior at infinity of $\Phi(\cdot)$ but we will only remark this on each respective section.

\subsection{Simple options}

We say that a simple option is a contingent claim $G^{\varepsilon}$ in the form $\Phi\left(S^{\varepsilon}(T)\right)$. Recall that in example 1.1, $\Phi(x)=\max (x-K, 0)$. If we for the moment assume that the payoff function is sufficiently smooth then formal calculations yield:

$$
\left.\frac{\partial \Pi^{\varepsilon}}{\partial \varepsilon}(0)\right|_{\varepsilon=0}=B(T)^{-1} E_{Q}\left[\Phi^{\prime}\left(S^{0}(T)\right) Z^{0}(T)\right],
$$

and

$$
\frac{\partial \Pi}{\partial \sigma}(0)=B(T)^{-1} E_{Q}\left[\Phi^{\prime}(S(T)) \frac{\partial S}{\partial \sigma}(T)\right]
$$

Hence, due to remark 2.1 we see that we only have to consider $\left.\frac{\partial \Pi^{\varepsilon}}{\partial \varepsilon}(0)\right|_{\varepsilon=0}$, since $\frac{\partial \Pi}{\partial \sigma}(0)$ can be obtained under A2 by just setting $\hat{\sigma}(\cdot)=1$ in $(3.2)$, i.e. under assumption $\mathrm{A} 2, \frac{\partial \Pi}{\partial \sigma}(0)=$ $\left.\frac{\partial \Pi^{\varepsilon}}{\partial \varepsilon}(0)\right|_{\varepsilon=0, \hat{\sigma}(\cdot)=1}$. An extension of this argument now gives us the following proposition, which generalizes the results in example 1.1 .

Proposition 3.1 For simple options, i.e. contingent claims with payoff $\Phi\left(S^{\varepsilon}(T)\right)$, we have that

$$
\left.\frac{\partial \Pi^{\varepsilon}}{\partial \varepsilon}(0)\right|_{\varepsilon=0}=\int_{0}^{T} E_{Q}\left[\mu(s, T) \hat{\sigma}\left(s, S^{0}(s)\right)\right] \sigma(s) d s,
$$

where $\mu(\cdot, T)$ is a positive adapted process that is independent of $\hat{\sigma}(\cdot, \cdot)$ but depends on $S^{0}(\cdot)$, $\Phi(\cdot)$ and its derivatives.

If we further assume $A 2$ we have

$$
\left.\frac{\partial \Pi^{\varepsilon}}{\partial \varepsilon}(0)\right|_{\varepsilon=0}=\frac{1}{T} \int_{0}^{T} \hat{\sigma}(t) d t \frac{\partial \Pi}{\partial \sigma}(0) .
$$


Note that although the two formulas in the above proposition look somewhat different, it is straightforward to rewrite the second formula in the general form of (3.3).

Proof. Let us start by considering the case where the payoff function $\Phi(\cdot)$ is infinitely continuously differentiable with bounded derivative. Using lemma 2.1 and the integration by parts formula we have from (3.2) that

$$
\begin{aligned}
\left.\frac{\partial \Pi^{\varepsilon}}{\partial \varepsilon}(0)\right|_{\varepsilon=0}= & -B(T)^{-1} E_{Q}\left[\Phi^{\prime}\left(S^{0}(T)\right) \mathcal{E}^{0}(T) \int_{0}^{T} \mathcal{E}^{0}(s)^{-1} \hat{\sigma}\left(s, S^{0}(s)\right) \sigma(s) d s\right. \\
& \left.\quad-\int_{0}^{T} \mathcal{E}^{0}(s)^{-1} \hat{\sigma}\left(s, S^{0}(s)\right) d V(s)\right] \\
= & -B(T)^{-1} E_{Q}\left[\int_{0}^{T} \Phi^{\prime}\left(S^{0}(T)\right) \mathcal{E}^{0}(T) \mathcal{E}^{0}(s)^{-1} \hat{\sigma}\left(s, S^{0}(s)\right) \sigma(s) d s\right] \\
& +B(T)^{-1} E_{Q}\left[\int_{0}^{T} D_{s}\left(\Phi^{\prime}\left(S^{0}(T)\right) \mathcal{E}^{0}(T)\right) \mathcal{E}^{0}(s)^{-1} \hat{\sigma}\left(s, S^{0}(s)\right) d s\right] .
\end{aligned}
$$

Moreover, from the identity $\mathcal{E}^{0}(\cdot)=S^{0}(\cdot) / S_{0}$, the chain rule, and the fact that $D_{t} S^{0}(s)=$ $\sigma(t) S^{0}(s) \mathbf{1}_{t \leq s}$, we also see that

$$
\begin{aligned}
D_{s}\left(\Phi^{\prime}\left(S^{0}(T)\right) \mathcal{E}^{0}(T)\right) & =\mathcal{E}^{0}(T) D_{s} \Phi^{\prime}\left(S^{0}(T)\right)+\Phi^{\prime}\left(S^{0}(T)\right) D_{s} \mathcal{E}^{0}(T) \\
& =\mathcal{E}^{0}(T) \Phi^{\prime \prime}\left(S^{0}(T)\right) \sigma(s) S^{0}(T)+\Phi^{\prime}\left(S^{0}(T)\right) \sigma(s) \mathcal{E}^{0}(T)
\end{aligned}
$$

Hence,

$$
\left.\frac{\partial \Pi^{\varepsilon}}{\partial \varepsilon}(0)\right|_{\varepsilon=0}=B(T)^{-1} \int_{0}^{T} E_{Q}\left[S^{0}(T) \Phi^{\prime \prime}\left(S^{0}(T)\right) \mathcal{E}^{0}(T) \mathcal{E}^{0}(s)^{-1} \sigma(s) \hat{\sigma}\left(s, S^{0}(s)\right)\right] d s,
$$

and consequently, it follows that

$$
\mu(s, T)=B(T)^{-1} E_{Q}\left[S^{0}(T)^{2} \Phi^{\prime \prime}\left(S^{0}(T)\right) \mid \mathcal{F}_{s}\right] S^{0}(s)^{-1} .
$$

In the case that where we assume A2 we have

$$
\left.\frac{\partial \Pi^{\varepsilon}}{\partial \varepsilon}(0)\right|_{\varepsilon=0}=B(T)^{-1} E_{Q}\left[S(T)^{2} \Phi^{\prime \prime}(S(T))\right] \sigma \int_{0}^{T} \hat{\sigma}(s) d s,
$$

and in particular

$$
\frac{\partial \Pi}{\partial \sigma}(0)=\left.\frac{\partial \Pi^{\varepsilon}}{\partial \varepsilon}(0)\right|_{\varepsilon=0, \hat{\sigma}(\cdot)=1}=B(T)^{-1} E_{Q}\left[S(T)^{2} \Phi^{\prime \prime}(S(T))\right] \sigma T,
$$

from which the relationship between the local Vega index and the classical Vega index is obtained.

Finally, we need to verify that (3.4) remain true for general payoff functions as in definition 3.1. It is a standard result, see e.g. Rudin (1976) theorem 7.17, that if there exists a sequence of functions $\left\{\Pi_{n}^{\varepsilon}(0)\right\}$ differentiable with respect to $\varepsilon$ such that $\Pi_{n}^{0}(0) \rightarrow \Pi^{0}(0)$ and the sequence of derivatives $\left\{\frac{\partial \Pi_{n}^{\varepsilon}}{\partial \varepsilon}(0)\right\}$ converges uniformly in $\varepsilon$, then $\frac{\partial \Pi^{\varepsilon}}{\partial \varepsilon}(0)=\lim _{n \rightarrow \infty} \frac{\partial \Pi_{n}^{\varepsilon}}{\partial \varepsilon}(0)$. 
We define $\Pi_{n}^{\varepsilon}(0)=B(T)^{-1} E_{Q}\left[\Phi_{n}\left(S^{\varepsilon}(T)\right)\right]$, where we let $\left\{\Phi_{n}(\cdot)\right\}$ be a sequence of infinitely continuously differentiable functions with bounded derivatives, i.e. in $C_{b}^{\infty}$. Given assumption A1, the payoff $\Phi\left(S^{\varepsilon}(T)\right)$ is square integrable for any $\varepsilon$, which is identical to saying that the payoff function $\Phi(\cdot)$ is in $L^{2}\left(\nu^{\varepsilon}\right)$ where $\nu^{\varepsilon}$ is the measure defined by $\nu^{\varepsilon}(A)=Q\left(S^{\varepsilon}(T) \in A\right)$. Furthermore, the corresponding density function, denoted $f^{\varepsilon}(\cdot)$, exists and is smooth, and one obtains (after a slight modification of the proof of Theorem 3.1 in Taniguchi (1985)) that if we define the function

$$
\tilde{f}(x)=\left\{\begin{array}{cl}
C \exp \left(-C[\ln (x)]^{2}\right) & ; x>x_{0} \\
C_{1} & ; x \leq x_{0}
\end{array}\right.
$$

then there exist constants $C, C_{1}$, and $x_{0}$ independent of $\varepsilon$ such that $f^{\varepsilon}(\cdot) \leq \tilde{f}(\cdot)$ for all $\varepsilon$. Now, defining the measure $\tilde{\nu}$ by $\tilde{\nu}(A)=\int_{A} \tilde{f}(x) d x$ we see that $\Phi(\cdot) \in L^{2}(\tilde{\nu}) \Rightarrow \Phi(\cdot) \in L^{2}\left(\nu^{\varepsilon}\right)$. Consequently, since the space $C_{b}^{\infty}$ is a dense subspace of $L^{2}(\tilde{\nu})$ we can always chose a sequence $\Phi_{n}(\cdot) \rightarrow \Phi(\cdot)$ in $L^{2}(\tilde{\nu})$ (and therefore also in $L^{2}\left(\nu^{\varepsilon}\right)$ for any $\varepsilon$ ). Trivially, we have that

$$
\begin{aligned}
\lim _{n \rightarrow \infty}\left|\Pi_{n}^{0}(0)-\Pi^{0}(0)\right| & \leq \lim _{n \rightarrow \infty} B(T)^{-1}\left(\int_{0}^{\infty}\left(\Phi_{n}(x)-\Phi(x)\right)^{2} f^{0}(x) d x\right)^{\frac{1}{2}} \\
& \leq B(T)^{-1} \lim _{n \rightarrow \infty}\left(\int_{0}^{\infty}\left(\Phi_{n}(x)-\Phi(x)\right)^{2} \tilde{f}(x) d x\right)^{\frac{1}{2}} \\
& =0 .
\end{aligned}
$$

By using the integration by parts on (3.2) we have that

$$
\frac{\partial \Pi_{n}^{\varepsilon}}{\partial \varepsilon}(0)=B(T)^{-1} E_{Q}\left[\Phi_{n}^{\prime}\left(S^{\varepsilon}(T)\right) Z^{\varepsilon}(T)\right]=B(T)^{-1} E_{Q}\left[\Phi_{n}\left(S^{\varepsilon}(T)\right) H^{\varepsilon}(T)\right],
$$

for some random variable $H^{\varepsilon}(T) \in L^{p}(\Omega, \mathcal{F}, Q)$ uniformly in $\varepsilon$ and for all $p>1$. Consequently, with $\|\cdot\|_{2}$ denoting the norm in $L^{2}(\Omega, \mathcal{F}, Q)$ we get

$$
\begin{aligned}
& \lim _{n \rightarrow \infty} \sup _{\varepsilon}\left|\frac{\partial \prod_{n}^{\varepsilon}}{\partial \varepsilon}(0)-B(T)^{-1} E_{Q}\left[\Phi\left(S^{\varepsilon}(T)\right) H^{\varepsilon}(T)\right]\right| \\
& \quad \leq \lim _{n \rightarrow \infty} \sup _{\varepsilon} B(T)^{-1}\left(\int_{0}^{\infty}\left(\Phi_{n}(x)-\Phi(x)\right)^{2} f^{\varepsilon}(x) d x\right)^{\frac{1}{2}}\left\|H^{\varepsilon}(T)\right\|_{2} \\
& \quad \leq B(T)^{-1} \lim _{n \rightarrow \infty}\left(\int_{0}^{\infty}\left(\Phi_{n}(x)-\Phi(x)\right)^{2} \tilde{f}(x) d x\right)^{\frac{1}{2}} \sup _{\varepsilon}\left\|H^{\varepsilon}(T)\right\|_{2} \\
& \quad=0 .
\end{aligned}
$$

This proves that the expression $\left.\frac{\partial \Pi^{\varepsilon}}{\partial \varepsilon}(0)\right|_{\varepsilon=0}$ is well defined and that in the particular case where we assume A2, we get: $\left.\frac{\partial \Pi^{\varepsilon}}{\partial \varepsilon}(0)\right|_{\varepsilon=0}=\left.\lim _{n \rightarrow \infty} \frac{\partial \Pi_{n}^{\varepsilon}}{\partial \varepsilon}(0)\right|_{\varepsilon=0}=\lim _{n \rightarrow \infty} \frac{1}{T} \int_{0}^{T} \hat{\sigma}(t) d t \frac{\partial \Pi_{n}}{\partial \sigma}(0)=$ $\frac{1}{T} \int_{0}^{T} \hat{\sigma}(t) d t \frac{\partial \Pi}{\partial \sigma}(0)$. To finish the proof in the general case we need to define the adapted process 
$\mu(\cdot, T)$ and show that it is a.s. positive. For this let us write $(3.4)$ as:

$$
\left.\frac{\partial \Pi_{n}^{\varepsilon}}{\partial \varepsilon}(0)\right|_{\varepsilon=0}=B(T)^{-1} \int_{0}^{T} E_{Q}\left[E_{Q}\left[S^{0}(T)^{2} \Phi_{n}^{\prime \prime}\left(S^{0}(T)\right) \mid S^{0}(s)\right] S^{0}(s)^{-1} \hat{\sigma}\left(s, S^{0}(s)\right)\right] \sigma(s) d s .
$$

We need to show that $E_{Q}\left[S^{0}(T)^{2} \Phi_{n}^{\prime \prime}\left(S^{0}(T)\right) \mid S^{0}(s)\right]:=\int_{0}^{\infty} y^{2} \Phi_{n}^{\prime \prime}(y) p_{T}\left(y \mid S^{0}(s)\right) d y$ converges to a positive adapted process. Here we let $p_{T}\left(y \mid S^{0}(s)\right)$ denote the conditional density of $S^{0}(T)$ given $S^{0}(s)$. Using integration by parts and then passing to the limit we obtain

$$
\int_{0}^{\infty} y^{2} \Phi_{n}^{\prime \prime}(y) p_{T}\left(y \mid S^{0}(s)\right) d y \rightarrow \int_{0}^{\infty} y^{2} p_{T}\left(y \mid S^{0}(s)\right) d \varsigma(y) \quad Q \text {-a.s. }
$$

The right hand side is positive and equal to $E_{Q}\left[S^{0}(T)^{2} \Phi^{\prime \prime}\left(S^{0}(T)\right) \mid S^{0}(s)\right]$ in the formal sense of (3.1). Therefore the positive adapted process $\mu(\cdot, T)$ is defined just as before.

Remark 3.1 Note that the above formulas are satisfied in greater generality. Under the assumption A2 we only need $\Phi(\cdot)$ to be an element of $L^{2}(\vartheta)$ where $\vartheta$ is a measure dominating all the measures $\nu^{\varepsilon}$. In particular, if $\Phi(\cdot)$ is any measurable function with polynomial growth at infinity, the relationship between the local Vega and the classical Vega index is maintained. In the general case one could also allow some flexibility for $\Phi(\cdot)$ but then the expression of the local Vega index starts to depend on the derivatives of $\hat{\sigma}(t, \cdot)$.

By considering the general case, assumption A1, we obtain a little bit more information about the local volatility $\hat{\sigma}(\cdot, \cdot)$ than what could be extracted from example 1.1. For instance, the expression (3.3) also says that there is a trade-off between the size of volatility and the permissible amount of mis-specification of it. In other words, the local Vega index will be the same in the following two cases: First, one allows $\hat{\sigma}(\cdot, \cdot)$ to be big when $\sigma(\cdot)$ is small and second one allows $\sigma(\cdot)$ to be big when $\hat{\sigma}(\cdot, \cdot)$ is small.

A natural question to pose is whether the local Vega index is positive or negative. For the sake of simplicity let us assume A2. It is easily verified that for simple options $\frac{\partial \Pi}{\partial \sigma}(0) \geq 0$, and therefore a sufficient condition for $\left.\frac{\partial \Pi^{\varepsilon}}{\partial \varepsilon}(0)\right|_{\varepsilon=0} \geq 0$ is that $\int_{0}^{T} \hat{\sigma}(t) d t \geq 0$. Furthermore, this also implies that $\Pi^{\varepsilon}(0) \geq \Pi(0)$ for $\varepsilon$ small enough.

Now, let us try to answer the following question: when is the price $\Pi^{1}(0)$ greater or equal than $\Pi(0)$. Note that the first price corresponds to the case where the stock has the volatility $\sigma+\hat{\sigma}(\cdot)$, while the second price corresponds to the case where the stock has the volatility $\sigma$. As shown in El Karoui et al. $(1998)$ a condition for $\Pi^{1}(0) \geq \Pi(0)$ is that $0 \leq \int_{0}^{T}\left[2 \sigma \hat{\sigma}(t)+\hat{\sigma}(t)^{2}\right] d t$. We will hint that this is only a sufficient condition and present an equivalent condition by using the concept of the local Vega index.

Following the proof of proposition 3.1 we easily find that

$$
\frac{\partial \Pi^{\varepsilon}}{\partial \varepsilon}(0)=C^{\varepsilon} \int_{0}^{T}[\sigma+\varepsilon \hat{\sigma}(t)] \hat{\sigma}(t) d t .
$$


where the positive constant $C^{\varepsilon}=B(T)^{-1} E_{Q}\left[\Phi^{\prime \prime}\left(S^{\varepsilon}(T)\right) S^{\varepsilon}(T)^{2}\right]$. Moreover, it follows from the Fubini theorem that

$$
\begin{aligned}
\Pi^{1}(0)-\Pi(0) & =\int_{0}^{1} \frac{\partial \Pi^{\varepsilon}}{\partial \varepsilon}(0) d \varepsilon \\
& =\int_{0}^{1} C^{\varepsilon} d \varepsilon\left(\int_{0}^{T}[\sigma+\bar{C} \hat{\sigma}(t)] \hat{\sigma}(t) d t\right),
\end{aligned}
$$

where $\bar{C}=\int_{0}^{1} \varepsilon C^{\varepsilon} d \varepsilon / \int_{0}^{1} C^{\varepsilon} d \varepsilon$. Hence, we see that $0 \leq \int_{0}^{T}\left[\sigma \hat{\sigma}(t)+\bar{C} \hat{\sigma}(t)^{2}\right] d t$ if and only if $\Pi^{1}(0) \geq \Pi(0)$.

\section{$3.2 \quad$ Asian options}

We say that an Asian option is a contingent claim $G^{\varepsilon}$ in the form $\Phi\left(\int_{0}^{T} w(t) S^{\varepsilon}(t) d t\right)$. Here $w(\cdot)$ is a bounded measurable positive weight function, such that $w(t) \geq w_{0}>0$ in a neighborhood around zero. Note that in this case there exists no closed solution for the price of the option and therefore there is no straightforward way of computing the sensibility indices we are interested in.

Just as in the previous example we will start by assuming that $\Phi(\cdot)$ is infinitely continuously differentiable with bounded derivatives. The general case will then be obtained by density arguments. Moreover, it is straightforward to show that we can interchange the order of integration and derivation since all the operators involved are linear. Hence, formal calculations yield:

$$
\left.\frac{\partial \Pi^{\varepsilon}}{\partial \varepsilon}(0)\right|_{\varepsilon=0}=B(T)^{-1} E_{Q}\left[\Phi^{\prime}\left(\int_{0}^{T} w(t) S^{0}(t) d t\right) \int_{0}^{T} w(t) Z^{0}(v) d v\right],
$$

and

$$
\frac{\partial \Pi}{\partial \sigma}(0)=B(T)^{-1} E_{Q}\left[\Phi^{\prime}\left(\int_{0}^{T} w(t) S(t) d t\right) \int_{0}^{T} w(v) \frac{\partial S}{\partial \sigma}(v) d v\right] .
$$

Again we see that we only have to consider $\left.\frac{\partial \Pi^{\varepsilon}}{\partial \varepsilon}(0)\right|_{\varepsilon=0}$, since $\frac{\partial \Pi}{\partial \sigma}(0)$ can be obtained by assuming $\mathrm{A} 2$ and setting $\hat{\sigma}(\cdot)=1$, i.e. under $\mathrm{A} 2, \frac{\partial \Pi}{\partial \sigma}(0)=\left.\frac{\partial \Pi^{\varepsilon}}{\partial \varepsilon}(0)\right|_{\varepsilon=0, \hat{\sigma}(\cdot)=1}$.

Proposition 3.2 For Asian options, i.e. contingent claims with payoff $\Phi\left(\int_{0}^{T} w(t) S^{\varepsilon}(t) d t\right)$, we have that

$$
\left.\frac{\partial \Pi^{\varepsilon}}{\partial \varepsilon}(0)\right|_{\varepsilon=0}=\int_{0}^{T} E\left[\mu(s, T) \hat{\sigma}\left(s, S^{0}(s)\right)\right] \sigma(s) d s,
$$

where $\mu(\cdot, T)$ is a positive adapted process that is independent of $\hat{\sigma}(\cdot, \cdot)$ but depends on $\Phi(\cdot)$ and its derivatives, and on the past of $S^{0}(\cdot)$ and $w(\cdot)$.

Furthermore, if one assumes A2 then

$$
\left.\frac{\partial \Pi^{\varepsilon}}{\partial \varepsilon}(0)\right|_{\varepsilon=0}=\int_{0}^{T} \bar{\mu}(s, T) \hat{\sigma}(s) d s \frac{\partial \Pi}{\partial \sigma}(0),
$$


where

$$
\bar{\mu}(s, T)=\frac{E_{Q}\left[\Phi^{\prime \prime}\left(\int_{0}^{T} w(t) S(t) d t\right)\left(\int_{s}^{T} w(t) S(t) d t\right)^{2}\right]}{\int_{0}^{T} E_{Q}\left[\Phi^{\prime \prime}\left(\int_{0}^{T} w(t) S(t) d t\right)\left(\int_{s}^{T} w(t) S(t) d t\right)^{2}\right] d s},
$$

and $\bar{\mu}(\cdot, T)$ is a decreasing density function such that $\lim _{s \rightarrow T} \bar{\mu}(s, T)=0 .{ }^{1}$

It can be shown that the pair $\left(\int_{0}^{T} w(t) S(t) d t, \int_{s}^{T} w(t) S(t) d t\right)$ has a smooth joint density for all $s \in[0, T]$ and therefore the function $\bar{\mu}(\cdot, T)$ is well defined in the formal sense of (3.1).

Proof. We start by considering the case where the payoff function $\Phi(\cdot)$ is infinitely continuously differentiable with bounded derivatives. First note that

$$
\begin{aligned}
\left.\frac{\partial \Pi^{\varepsilon}}{\partial \varepsilon}(0)\right|_{\varepsilon=0} & =B(T)^{-1} \int_{0}^{T} E_{Q}\left[\Phi^{\prime}\left(\int_{0}^{T} w(t) S^{0}(t) d t\right) w(s) Z^{0}(s)\right] d s \\
& =-B(T)^{-1} \int_{0}^{T} E_{Q}\left[\Phi ^ { \prime } ( \int _ { 0 } ^ { T } w ( t ) S ^ { 0 } ( t ) d t ) \left(\int_{0}^{s} \mathcal{E}^{0}(u)^{-1} \sigma(u) \hat{\sigma}\left(u, S^{0}(u)\right) d u\right.\right. \\
& \left.\left.\quad-\int_{0}^{s} \mathcal{E}^{0}(u)^{-1} \hat{\sigma}\left(u, S^{0}(u)\right) d V(u)\right) \mathcal{E}^{0}(s)\right] w(s) d s \\
= & B(T)^{-1} \int_{0}^{T} \int_{0}^{s} E_{Q}\left[\Phi^{\prime \prime}\left(\int_{0}^{T} w(t) S^{0}(t) d t\right) \int_{u}^{T} w(v) \sigma(u) S^{0}(v) d v \mathcal{E}^{0}(s) \cdot\right. \\
& \left.\cdot \mathcal{E}^{0}(u)^{-1} \hat{\sigma}\left(u, S^{0}(u)\right)\right] d u w(s) d s .
\end{aligned}
$$

The last equality follows from the fact that by using the integration by parts formula, we see that

$$
E_{Q}\left[\Phi^{\prime}\left(\int_{0}^{T} w(t) S^{0}(t) d t\right) \mathcal{E}^{0}(s) \int_{0}^{s} \mathcal{E}^{0}(u)^{-1} \hat{\sigma}\left(u, S^{0}(u)\right) d V(u)\right]
$$

is equal to

$$
E_{Q}\left[\int_{0}^{s} D_{u}\left(\Phi^{\prime}\left(\int_{0}^{T} w(t) S^{0}(t) d t\right) \mathcal{E}^{0}(s)\right) \mathcal{E}^{0}(u)^{-1} \hat{\sigma}\left(u, S^{0}(u)\right) d u\right],
$$

where in addition

$$
\begin{aligned}
D_{u}\left(\Phi^{\prime}\left(\int_{0}^{T} w(t) S^{0}(t) d t\right) \mathcal{E}^{0}(s)\right)= & \Phi^{\prime}\left(\int_{0}^{T} w(t) S^{0}(t) d t\right) D_{u} \mathcal{E}^{0}(s) \\
& +\mathcal{E}^{0}(s) D_{u} \Phi^{\prime}\left(\int_{0}^{T} w(t) S^{0}(t) d t\right) \\
= & \Phi^{\prime}\left(\int_{0}^{T} w(t) S^{0}(t) d t\right) \sigma(u) \mathcal{E}^{0}(s) 1_{u \leq s} \\
& +\mathcal{E}^{0}(s) \Phi^{\prime \prime}\left(\int_{0}^{T} w(t) S^{0}(t) d t\right) \sigma(u) \int_{u}^{T} w(t) S^{0}(t) d t
\end{aligned}
$$

\footnotetext{
${ }^{1}$ From here and on we interpret $\frac{0}{0}=0$ for all the density functions.
} 
as $D_{u} \int_{0}^{T} w(t) S^{0}(t) d t=\int_{0}^{T} w(t) D_{u} S^{0}(t) d t=\int_{0}^{T} w(t) \sigma(u) S^{0}(t) \mathbf{1}_{u \leq t} d t=\sigma(u) \int_{u}^{T} w(t) S^{0}(t) d t$.

Now, by changing the order of integration we get the representation

$$
\begin{gathered}
\left.\frac{\partial \Pi^{\varepsilon}}{\partial \varepsilon}(0)\right|_{\varepsilon=0}=B(T)^{-1} \int_{0}^{T} E_{Q}\left[\Phi^{\prime \prime}\left(\int_{0}^{T} w(t) S^{0}(t) d t\right) \sigma(u) \mathcal{E}^{0}(u)^{-1} \hat{\sigma}\left(u, S^{0}(u)\right) \cdot\right. \\
\left.\cdot \int_{u}^{T} w(v) S^{0}(v) d v \int_{u}^{T} \mathcal{E}^{0}(s) w(s) d s\right] d u .
\end{gathered}
$$

This equation shows that the adapted positive process $\mu(\cdot, T)$ should be defined as

$$
\mu(u, T)=B(T)^{-1} S^{0}(u)^{-1} E_{Q}\left[\Phi^{\prime \prime}\left(\int_{0}^{T} w(t) S^{0}(t) d t\right)\left(\int_{u}^{T} w(v) S^{0}(v) d v\right)^{2} \mid \mathcal{F}_{u}\right]
$$

since by definition $\mathcal{E}^{0}(\cdot)=S^{0}(\cdot) / S_{0}$.

In order to show that the expression for $\left.\frac{\partial \Pi^{\varepsilon}}{\partial \varepsilon}(0)\right|_{\varepsilon=0}$ remains true for general payoff functions as in definition 3.1, one proceeds as in the proof of proposition 3.1. The properties needed are proved in Appendix B.

Finally, by assuming A2, one proves as before the relationship between the classical and local Vega index.

We observe that the relations are qualitatively similar to the ones in Proposition 3.1. Therefore similar remarks as those already made are valid for this case too. In particular, under assumption A2 one finds similar to the previous section that there exists a positive decreasing density function $\bar{\mu}_{\varepsilon}(\cdot, T)$ such that

$$
\begin{aligned}
\Pi^{1}(0)-\Pi(0) & =\int_{0}^{1} \int_{0}^{T} \bar{\mu}_{\varepsilon}(s, T) \hat{\sigma}(s)(\sigma+\varepsilon \hat{\sigma}(s)) d s d \varepsilon \\
& =\int_{0}^{T} C(s) \hat{\sigma}(s) \sigma+\bar{C}(s) \hat{\sigma}(s)^{2} d s
\end{aligned}
$$

for certain positive deterministic functions $C(\cdot)$ and $\bar{C}(\cdot)$. In Appendix A we show in particular that $\Pi^{1}(0) \geq \Pi(0)$ if $\hat{\sigma}(\cdot) \geq 0$.

By choosing particular forms for the weight function $w(\cdot)$ one can study properties of other Asian type options such as, for instance, discretely monitored Asian options. Needless to say, this is of great importance since every Asian option traded at the market is discretely monitored, i.e. in the form $\Phi\left(\sum_{i=1}^{n} h\left(t_{i}\right) S^{\varepsilon}\left(t_{i}\right)\right)$ rather than $\Phi\left(\int_{0}^{T} w(t) S^{\varepsilon}(t) d t\right)$. Note also that the weight function is easily extended to the multi dimensional case.

\subsection{Lookback options}

So far, we have treated somewhat smooth functionals of the underlying asset. Now we concentrate on the case of an option based on an irregular functional such as the maximum process. As a consequence, the techniques used in the previous section cannot be used in this case due to 
the lack of smoothness of the maximum process. In order to solve this problem without having to worry about other technicalities, we assume A2 throughout this section.

We say that a lookback option is a contingent claim whose payoff function $G^{\varepsilon}$ is in the form $\Phi\left(\sup _{0 \leq t \leq T} S^{\varepsilon}(t)\right)$ for some payoff function. (Actually it is also possible to define lookback options as contingent claims in the form $G^{\varepsilon}=\Phi\left(\inf _{0 \leq t \leq T} S^{\varepsilon}(t)\right)$, however, for notational simplicity we do not consider this case). This time, though, it is not obvious that we can interchange the order of integration and derivation since the running maximum process is highly path-dependent and non-smooth. Moreover, the problem is that we do not have a closed form expression for the derivative with respect to $\varepsilon$ of the running maximum process and therefore we cannot simply use formal calculations to obtain a relationship between the two approaches. However, as shown in Appendix A, we still have the inequality $\Pi^{\varepsilon}(0) \geq \Pi(0)$ if the local volatility change is of the form $\hat{\sigma}(\cdot) \geq 0$ and $\Phi^{\prime}(0) \geq 0$.

Proposition 3.3 For lookback options, i.e. contingent claims with payoff $\Phi\left(\sup _{0 \leq t \leq T} S^{\varepsilon}(t)\right)$, we have that

$$
\begin{aligned}
\frac{\partial \Pi}{\partial \sigma}(0) & =\left.\frac{\partial \Pi^{\varepsilon}}{\partial \varepsilon}(0)\right|_{\varepsilon=0, \hat{\sigma}(\cdot)=1} \\
\left.\frac{\partial \Pi^{\varepsilon}}{\partial \varepsilon}(0)\right|_{\varepsilon=0} & =\int_{0}^{T} \bar{\mu}(s, T) \hat{\sigma}(s) d s \frac{\partial \Pi}{\partial \sigma}(0)
\end{aligned}
$$

where the density function $\bar{\mu}(\cdot, T)$ is given by

$$
\bar{\mu}(s, T)=\frac{E_{Q}\left[\Phi^{\prime}\left(\sup _{0 \leq t \leq T} S(t)\right) \sup _{0 \leq t \leq T} S(t)\left(\frac{2 r}{\sigma} 1_{s \leq \tau}+X\right)\right]}{\int_{0}^{T} E_{Q}\left[\Phi^{\prime}\left(\sup _{0 \leq t \leq T} S(t)\right) \sup _{0 \leq t \leq T} S(t)\left(\frac{2 r}{\sigma} 1_{s \leq \tau}+X\right)\right] d s} .
$$

The random time $\tau$ is implicitly defined by the relation $\sup _{0 \leq t \leq T} S(t)=S(\tau)$ and $X$ is an appropriate random variable that belongs to $L^{p}(\Omega, \mathcal{F}, Q)$ for any $p$. Furthermore, if $\Phi(\cdot)$ is monotone then $\mu(\cdot, T)$ is decreasing and if $\Phi^{\prime}(0) \geq 0$ then $\lim _{s \rightarrow T} \mu(s, T) \geq 0$.

Before giving the proof we present an auxiliary lemma. For the proof, see Seshadri (1988).

Lemma 3.4 Let $a$ and $c$ be real numbers. Suppose that $W(\cdot)$ is a Brownian motion and let the process $X(\cdot)$ be defined by:

$$
X(t)=\sup _{0 \leq s \leq t}(a W(s)+c s) .
$$

Let $U$ be a centered Gaussian stochastic variable with variance $t$, and let $F$ be an exponential stochastic variable with parameter $(2 t)^{-1}$ independent of $U$. Put

$$
Y=\frac{1}{2}\left(a U+c t+\sqrt{a^{2} F+(a U+c t)^{2}}\right) .
$$

Then the pairs $(W(t), X(t))$ and $(U, Y)$ have identical distributions 
Now we are ready to do the proof of proposition 3.3.

Proof. In the proof we will assume directly that the payoff function $\Phi(\cdot)$ is in the form of definition 3.1. Let us start by defining the stochastic integral $M^{\varepsilon}(\cdot):=\int_{0}^{\cdot}(\sigma+\varepsilon \hat{\sigma}(s)) d V(s)$ and the quadratic variation process $\left\langle M^{\varepsilon}\right\rangle .=\int_{0}^{\cdot}(\sigma+\varepsilon \hat{\sigma}(s))^{2} d s$. It follows from (1.6) that

$$
\sup _{0 \leq t \leq T} S^{\varepsilon}(t)=S_{0} \exp \left(\sup _{0 \leq t \leq T} A^{\varepsilon}(t)\right)
$$

with $A^{\varepsilon}(t)=r t-\frac{1}{2}\left\langle M^{\varepsilon}\right\rangle_{t}+M^{\varepsilon}(t)$. Moreover, since $t=\int_{0}^{\left\langle M^{\varepsilon}\right\rangle_{t}^{-1}}(\sigma+\varepsilon \hat{\sigma}(s))^{2} d s$ implicit derivation with respect to $t$ yields that $\frac{d\left\langle M^{\varepsilon}\right\rangle_{t}^{-1}}{d t}=\left(\sigma+\varepsilon \hat{\sigma}\left(\left\langle M^{\varepsilon}\right\rangle_{t}^{-1}\right)\right)^{-2}$. Consequently we have

$$
\begin{aligned}
A^{\varepsilon}\left(\left\langle M^{\varepsilon}\right\rangle_{t}^{-1}\right) & =\int_{0}^{t} \frac{r}{\left(\sigma+\varepsilon \hat{\sigma}\left(\left\langle M^{\varepsilon}\right\rangle_{s}^{-1}\right)\right)^{2}} d s-\frac{1}{2} t+M^{\varepsilon}\left(\left\langle M^{\varepsilon}\right\rangle_{t}^{-1}\right) \\
& =\int_{0}^{t} h^{\varepsilon}(s) d s+\left(\frac{r}{\sigma^{2}}-\frac{1}{2}\right) t+\bar{B}(t),
\end{aligned}
$$

where by definition $\bar{B}(t)=M^{\varepsilon}\left(\left\langle M^{\varepsilon}\right\rangle_{t}^{-1}\right)$ and $h^{\varepsilon}(s)=-\frac{r \varepsilon \hat{\sigma}\left(\left\langle M^{\varepsilon}\right\rangle^{-1}\right)\left(2 \sigma+\varepsilon \hat{\sigma}\left(\left\langle M^{\varepsilon}\right\rangle_{s}^{-1}\right)\right)}{\sigma^{2}\left(\sigma+\epsilon \hat{\sigma}\left(\left\langle M^{\varepsilon}\right\rangle_{s}^{-1}\right)\right)^{2}}$. In particular, note that $\bar{B}(\cdot)$ is a $Q$-Brownian motion, see e.g. Karatzas and Shreve (1988) page 174 . Therefore the problem is reduced to finding the derivative with respect to $\varepsilon$ of

$$
\begin{aligned}
\Pi^{\varepsilon}(0) & =B(T)^{-1} E_{Q}\left[\Phi\left(\sup _{0 \leq t \leq T} S^{\varepsilon}(t)\right)\right] \\
& =B(T)^{-1} E_{Q}\left[\Phi\left(S_{0} \exp \left(\sup _{0 \leq t \leq T} \int_{0}^{\left\langle M^{\varepsilon}\right\rangle_{t}} h^{\varepsilon}(s) d s+c\left\langle M^{\varepsilon}\right\rangle_{t}+\bar{B}\left(\left\langle M^{\varepsilon}\right\rangle_{t}\right)\right)\right)\right] \\
& =B(T)^{-1} E_{Q}\left[\Phi\left(S_{0} \exp \left(\sup _{0 \leq t \leq\left\langle M^{\varepsilon}\right\rangle_{T}} \int_{0}^{t} h^{\varepsilon}(s) d s+c t+\bar{B}(t)\right)\right)\right],
\end{aligned}
$$

where we have set $c=\frac{r}{\sigma^{2}}-\frac{1}{2}$. Now using the Girsanov theorem we see that the expectation in the above expression is equal to

$$
E_{Q^{\varepsilon}}\left[\frac{d Q}{d Q^{\varepsilon}} \Phi\left(S_{0} \exp \left(\sup _{0 \leq t \leq\left\langle M^{\varepsilon}\right\rangle_{T}} c t+V^{\varepsilon}(t)\right)\right)\right]
$$

where $V^{\varepsilon}(\cdot):=\int_{0}^{\cdot} h^{\varepsilon}(s) d s+\bar{B}(\cdot)$ is a $Q^{\varepsilon}$-Brownian motion and

$$
\frac{d Q}{d Q^{\varepsilon}}=\exp \left(-\frac{1}{2} \int_{0}^{\left\langle M^{\varepsilon}\right\rangle_{T}} h^{\varepsilon}(t)^{2} d t-\int_{0}^{\left\langle M^{\varepsilon}\right\rangle_{T}} h^{\varepsilon}(t) d V^{\varepsilon}(t)\right)
$$

Hence, in order to find the derivative of (3.5) we have to compute the limit when $\varepsilon \rightarrow 0$ of:

$$
\varepsilon^{-1}\left(E_{Q^{\varepsilon}}\left[\frac{d Q}{d Q^{\varepsilon}} \Phi\left(S_{0} \exp \left(\sup _{0 \leq t \leq\left\langle M^{\varepsilon}\right\rangle_{T}} c t+V^{\varepsilon}(t)\right)\right)\right]-E_{Q}\left[\Phi\left(\sup _{0 \leq t \leq T} S(t)\right)\right]\right) .
$$


By construction $V^{\varepsilon}(\cdot)$ will remain a $Q^{\varepsilon}$-Brownian motion for all values of $\varepsilon$, and therefore we may just as well consider

$$
\varepsilon^{-1}\left(E\left[\exp \left(-\frac{1}{2} \int_{0}^{\left\langle M^{\varepsilon}\right\rangle_{T}} h^{\varepsilon}(t)^{2} d t-\int_{0}^{\left\langle M^{\varepsilon}\right\rangle_{T}} h^{\varepsilon}(t) d W(t)\right) \Phi\left(Z^{\varepsilon}\right)\right]-E\left[\Phi\left(Z^{0}\right)\right]\right),
$$

where $Z^{\varepsilon}:=S_{0} \exp \left(\sup _{0 \leq t \leq\left\langle M^{\varepsilon}\right\rangle_{T}} c t+W(t)\right)$ and $W(\cdot)$ is a canonical Brownian motion independent of $V(\cdot), V^{\varepsilon}(\cdot)$, and $\bar{B}(\cdot)$. Note that it follows from a change of variables that $Z^{0} \stackrel{\mathcal{L}}{=} \sup _{0 \leq t \leq T} S(t)$. Now, we rewrite the above expression as

$$
\begin{aligned}
& \varepsilon^{-1} E\left[\left(\exp \left(-\frac{1}{2} \int_{0}^{\left\langle M^{\varepsilon}\right\rangle_{T}} h^{\varepsilon}(t)^{2} d t-\int_{0}^{\left\langle M^{\varepsilon}\right\rangle_{T}} h^{\varepsilon}(t) d W(t)\right)-1\right) \Phi\left(Z^{\varepsilon}\right)\right] \\
& +\varepsilon^{-1}\left(E\left[\Phi\left(Z^{\varepsilon}\right)\right]-E\left[\Phi\left(Z^{0}\right)\right]\right),
\end{aligned}
$$

where as before $c=\frac{r}{\sigma^{2}}-\frac{1}{2}$. According to lemma 3.4, we have for all $\varepsilon$ the alternative characterization:

$$
Z^{\varepsilon} \stackrel{\mathcal{L}}{=} S_{0} \exp \left(\frac{1}{2}\left(U^{\varepsilon}+c\left\langle M^{\varepsilon}\right\rangle_{T}+\sqrt{F^{\varepsilon}+\left(U^{\varepsilon}+c\left\langle M^{\varepsilon}\right\rangle_{T}\right)^{2}}\right)\right)=: Y^{\varepsilon}
$$

where $U^{\varepsilon}=\sqrt{\left\langle M^{\varepsilon}\right\rangle_{T}} \cdot A$ with $A \sim N(0,1)$, and $F^{\varepsilon}=2\left\langle M^{\varepsilon}\right\rangle_{T} \cdot B$ with $B \sim \operatorname{exp~(1).~From~this~}$ representation though one easily obtains that the second limit exists and is given by the usual derivative

$$
\left.\frac{\partial}{\partial \varepsilon} E\left[\Phi\left(Z^{\varepsilon}\right)\right]\right|_{\varepsilon=0}=\left.\frac{\partial}{\partial \varepsilon} E\left[\Phi\left(Y^{\varepsilon}\right)\right]\right|_{\varepsilon=0}
$$

Now, we would like to interchange order of derivation and integration and to justify this we recall that a convex function (our payoff function) is almost everywhere differentiable. Moreover, according to (3.7) the argument of $\Phi(\cdot)$ has a density and therefore the above derivative is given by:

$$
\begin{gathered}
E\left[\Phi ^ { \prime } ( Y ^ { 0 } ) Y ^ { 0 } \frac { 1 } { 2 } \int _ { 0 } ^ { T } \hat { \sigma } ( s ) d s \left\{T^{-\frac{1}{2}} A+2 \sigma c+\left(2 \sigma^{2} T B+\left(\sigma \sqrt{T} A+c \sigma^{2} T\right)^{2}\right)^{-\frac{1}{2}}\right.\right. \\
\left.\left.\cdot\left(2 \sigma B+\left(\sigma \sqrt{T} A+c \sigma^{2} T\right)\left(T^{-\frac{1}{2}} A+2 \sigma c\right)\right)\right\}\right]
\end{gathered}
$$

which can be written as $E\left[\hat{X} \Phi^{\prime}\left(Y^{0}\right) Y^{0}\right] \int_{0}^{T} \hat{\sigma}(s) d s$, for some appropriate random variable $\hat{X}$ that does not depend on $\hat{\sigma}(\cdot)$. Finally, by using $(3.7)$ and the relation $Z^{0} \stackrel{\mathcal{L}}{=} \sup _{0 \leq t \leq T} S(t)$, we conclude that the second limit is given by

$$
E_{Q}\left[X \Phi^{\prime}\left(\sup _{0 \leq t \leq T} S(t)\right) \sup _{0 \leq t \leq T} S(t)\right] \int_{0}^{T} \hat{\sigma}(s) d s
$$

where $X$ is an appropriate copy of $\hat{X}$ in the space $(\Omega, \mathcal{F}, Q)$. 
The limit of the first term in (3.6) exists and is equal to

$$
E\left[\int_{0}^{\sigma^{2} T} \frac{2 r \hat{\sigma}\left(s / \sigma^{2}\right)}{\sigma^{3}} d W(s) \Phi\left(Z^{0}\right)\right]
$$

The way to obtain the result is to use the relation $e^{x}-1=x+e^{x_{0}} x^{2} / 2$ for some $x_{0} \in[0, x]$, and noting that $\varepsilon^{-1} \int_{0}^{\left\langle M^{\varepsilon}\right\rangle_{T}} h^{\varepsilon}(t)^{2} d t \rightarrow 0$ and $\Phi\left(Z^{\varepsilon}\right) \rightarrow \Phi\left(Z^{0}\right)$ when $\varepsilon \rightarrow 0$. Here the convergence is in the sense of almost surely, and also in $L^{2}(\Omega)$. Therefore, we only have to verify that $\left|-\varepsilon^{-1} \int_{0}^{\left\langle M^{\varepsilon}\right\rangle_{T}} h^{\varepsilon}(t) d W(t)-\int_{0}^{\sigma^{2} T} \frac{2 r \hat{\sigma}\left(s / \sigma^{2}\right)}{\sigma^{3}} d W(s)\right| \rightarrow 0$ a.s. when $\varepsilon \rightarrow 0$, since higher order terms vanishes. Inserting the expression for $h^{\varepsilon}(\cdot)$ and doing some algebra we find that

$$
-\varepsilon^{-1} \int_{0}^{\left\langle M^{\varepsilon}\right\rangle_{T}} h^{\varepsilon}(t) d W(t)-\int_{0}^{\sigma^{2} T} \frac{2 r \hat{\sigma}\left(s / \sigma^{2}\right)}{\sigma^{3}} d W(s)
$$

equals

$$
\int_{0}^{\left\langle M^{\varepsilon}\right\rangle_{T}} \frac{2 r\left[\hat{\sigma}\left(\left\langle M^{\varepsilon}\right\rangle_{s}^{-1}\right)-\hat{\sigma}\left(s / \sigma^{2}\right)\right]}{\sigma\left(\sigma+\varepsilon \hat{\sigma}\left(\left\langle M^{\varepsilon}\right\rangle_{s}^{-1}\right)\right)^{2}} d W(s)-\varepsilon^{-1} \int_{\sigma^{2} T}^{\left\langle M^{\varepsilon}\right\rangle_{T}} h^{\varepsilon}(s) d W(s)+\mathcal{O}(\varepsilon)
$$

This expression though, goes to zero almost surely if $\hat{\sigma}(\cdot)$ is a continuous function since $\left\langle M^{\varepsilon}\right\rangle_{s}^{-1} \rightarrow$ $s / \sigma^{2}$. Now, consider the case where $\hat{\sigma}(\cdot) \in L^{2}\left(\left[0, T^{*}\right]\right)$. It is easily shown that $(3.8)$ is true if we can prove that $\lim _{\varepsilon \rightarrow 0} \int_{0}^{\left\langle M^{\varepsilon}\right\rangle_{T}}\left[\hat{\sigma}\left(\left\langle M^{\varepsilon}\right\rangle_{s}^{-1}\right)-\hat{\sigma}\left(s / \sigma^{2}\right)\right]^{2} d s=0$. First, note that we can always find a sequence of continuously differentiable functions $\left\{f_{\alpha}(\cdot)\right\}$ such that $\int_{0}^{T}\left[\hat{\sigma}(s)-f_{\alpha}(s)\right]^{2} d s \leq$ $\alpha$ for any $\alpha$. Therefore

$$
\begin{aligned}
\int_{0}^{\left\langle M^{\varepsilon}\right\rangle_{T}}\left[\hat{\sigma}\left(\left\langle M^{\varepsilon}\right\rangle_{s}^{-1}\right)-\hat{\sigma}\left(s / \sigma^{2}\right)\right]^{2} d s \leq & C\left(\int_{0}^{\left\langle M^{\varepsilon}\right\rangle_{T}}\left[\hat{\sigma}\left(\left\langle M^{\varepsilon}\right\rangle_{s}^{-1}\right)-f_{\alpha}\left(\left\langle M^{\varepsilon}\right\rangle_{s}^{-1}\right)\right]^{2} d s\right. \\
& +\int_{0}^{\left\langle M^{\varepsilon}\right\rangle_{T}}\left[f_{\alpha}\left(s / \sigma^{2}\right)-f_{\alpha}\left(\left\langle M^{\varepsilon}\right\rangle_{s}^{-1}\right)\right]^{2} d s \\
& \left.+\int_{0}^{\left\langle M^{\varepsilon}\right\rangle_{T}}\left[f_{\alpha}\left(s / \sigma^{2}\right)-\hat{\sigma}\left(s / \sigma^{2}\right)\right]^{2} d s\right) \\
\leq & C\left(2 \alpha+\int_{0}^{\left\langle M^{\varepsilon}\right\rangle_{T}}\left[f_{\alpha}\left(s / \sigma^{2}\right)-f_{\alpha}\left(\left\langle M^{\varepsilon}\right\rangle_{s}^{-1}\right)\right]^{2} d s\right),
\end{aligned}
$$

for some constant $C$, from which (3.8) follows.

Finally, we want to rewrite (3.8) using the integration by parts formula in order to identify a density function just like in the case of the previous studied contingent claims. However, note that after a time change and a change of probability space we can express (3.8) as

$$
E_{Q}\left[\int_{0}^{T} \frac{2 r \hat{\sigma}(u)}{\sigma} d V(u) \Phi\left(\sup _{0 \leq s \leq T} S(s)\right)\right] .
$$


Since $\Phi(\cdot)$ is a convex function with bounded derivatives it is also a Lipschitz function. Moreover, the maximum of the stock price has a density and belongs to the space $\mathbb{D}^{1,2}$. Consequently, $\Phi\left(\sup _{0 \leq s \leq T} S(s)\right) \in \mathbb{D}^{1,2}$, see Nualart (1995) for details, and the usual chain rule applies. Hence, we can rewrite $(3.8)$ as

$$
\frac{2 r}{\sigma} E_{Q}\left[\int_{0}^{T} \hat{\sigma}(u) \Phi^{\prime}\left(\sup _{0 \leq s \leq T} S(s)\right) D_{u} \sup _{0 \leq s \leq T} S(s) d u\right] .
$$

The Malliavin derivative $D_{u} \sup _{0 \leq s \leq T} S(s)=\sigma \sup _{0 \leq s \leq T} S(s) \mathbf{1}_{u \leq \tau}$ where $\tau$ is the random time where the maximum is achieved, see for instance Bermin (1998a). Hence, we get the expression

$$
\left.\frac{\partial \Pi^{\varepsilon}}{\partial \varepsilon}(0)\right|_{\varepsilon=0}=B(T)^{-1} \int_{0}^{T} E_{Q}\left[\Phi^{\prime}\left(\sup _{0 \leq t \leq T} S(t)\right) \sup _{0 \leq t \leq T} S(t)\left(\frac{2 r}{\sigma} \mathbf{1}_{u \leq \tau}+X\right)\right] \hat{\sigma}(u) d u .
$$

Now, if we repeat the above calculations for the case of the derivative $\frac{\partial \Pi}{\partial \sigma}(0)$, we find that

$$
\frac{\partial \Pi}{\partial \sigma}(0)=\left.\frac{\partial \Pi^{\varepsilon}}{\partial \varepsilon}(0)\right|_{\varepsilon=0, \hat{\sigma}(\cdot)=1}
$$

from which we conclude that there exists a density function $\bar{\mu}(\cdot, T)$ as stated in the proposition.

In order to verify the decreasing property of $\bar{\mu}(\cdot, T)$ whenever $\Phi(\cdot)$ is monotone, we start by considering the case where the payoff function $\Phi(\cdot)$ is increasing. The first derivative, which exists almost everywhere, then is non-negative. Consequently,

$$
E_{Q}\left[\Phi^{\prime}\left(\sup _{0 \leq t \leq T} S(t)\right) \sup _{0 \leq t \leq T} S(t) \frac{2 r}{\sigma} \mathbf{1}_{s \leq \tau}\right]
$$

is decreasing since the set over which we integrate, i.e. $\{s \leq \tau\}$, is decreasing. For the case where the payoff function $\Phi(\cdot)$ is decreasing we note that $-\Phi^{\prime}(\cdot)$ is non-negative and the same arguments apply thanks to the denominator in the expression for $\bar{\mu}(\cdot, T)$. Moreover, if we consider a local volatility change $\hat{\sigma}(\cdot) \geq 0$, then according to Appendix $A,\left.\frac{\partial \Pi^{\varepsilon}}{\partial \varepsilon}(0)\right|_{\varepsilon=0}$ and consequently also $\frac{\partial \Pi}{\partial \sigma}(0)$, are non-negative. As a conclusion we find that the density function $\bar{\mu}(\cdot, T)$ is non-negative as well.

To get a little bit more intuition of the way the local Vega index and the classical Vega index are related one to the other, we present the following result.

Corollary 3.5 For lookback options we have the alternative characterization

$$
\begin{aligned}
\left.\frac{\partial \Pi^{\varepsilon}}{\partial \varepsilon}(0)\right|_{\varepsilon=0}= & \frac{1}{T} \int_{0}^{T} \hat{\sigma}(t) d t \frac{\partial \Pi}{\partial \sigma}(0)+\frac{2 r}{\sigma^{2}} e^{-r T} . \\
& \cdot E_{Q}\left[\Phi\left(\sup _{0 \leq s \leq T} S(s)\right)\left(\int_{0}^{T} \hat{\sigma}(t) d V(t)-V(T) \frac{1}{T} \int_{0}^{T} \hat{\sigma}(t) d t\right)\right] .
\end{aligned}
$$


In particular if $\hat{\sigma}(\cdot)$ is differentiable, then

$$
\begin{aligned}
\left.\frac{\partial \Pi^{\varepsilon}}{\partial \varepsilon}(0)\right|_{\varepsilon=0}= & \frac{1}{T} \int_{0}^{T} \hat{\sigma}(t) d t \frac{\partial \Pi}{\partial \sigma}(0)+\frac{2 r}{\sigma^{2}} e^{-r T} . \\
& \cdot E_{Q}\left[\Phi\left(\sup _{0 \leq s \leq T} S(s)\right)\left(V(T) \frac{1}{T} \int_{0}^{T}(\hat{\sigma}(T)-\hat{\sigma}(t)) d t-\int_{0}^{T} \frac{d \hat{\sigma}}{d t}(t) V(t) d t\right)\right] .
\end{aligned}
$$

Proof. From the previous proof we have that

$$
\begin{aligned}
\left.\frac{\partial \Pi^{\varepsilon}}{\partial \varepsilon}(0)\right|_{\varepsilon=0}= & B(T)^{-1} E_{Q}\left[\int_{0}^{T} \frac{2 r \hat{\sigma}(s)}{\sigma^{2}} d V(s) \Phi\left(\sup _{0 \leq t \leq T} S(t)\right)\right] \\
& +\int_{0}^{T} E\left[X \Phi^{\prime}\left(\sup _{0 \leq t \leq T} S(t)\right) \sup _{0 \leq t \leq T} S(t)\right] \hat{\sigma}(s) d s,
\end{aligned}
$$

which together with the property $\frac{\partial \Pi}{\partial \sigma}(0)=\left.\frac{\partial \Pi^{\varepsilon}}{\partial \varepsilon}(0)\right|_{\varepsilon=0, \hat{\sigma}(\cdot)=1}$ yield the first part of the proof. The second part then follows by using the integration by parts formula for stochastic integrals.

Note that this last statement shows the dependence on the way the anticipated volatility structure changes. For example if one considers $\hat{\sigma}(t)=\sigma_{1} \mathbf{1}(t \leq T / 2)+\sigma_{2} \mathbf{1}(t>T / 2)$, one finds that the local Vega index is given by

$$
\left.\frac{\partial \Pi^{\varepsilon}}{\partial \varepsilon}(0)\right|_{\varepsilon=0}=\frac{\sigma_{2}+\sigma_{1}}{2} \frac{\partial \Pi}{\partial \sigma}(0)+\frac{r e^{-r T}}{\sigma^{2}}\left(\sigma_{1}-\sigma_{2}\right) E_{Q}\left[\Phi\left(\sup _{0 \leq s \leq T} S(s)\right)\left(V\left(\frac{T}{2}\right)-\frac{V(T)}{2}\right)\right] .
$$

The second term could be positive or negative according to what the values for $\sigma_{1}$ and $\sigma_{2}$ are, which shows a clear dependence of the behavior of the price on the modulus of continuity of the local change in volatility. Note that if we assume $\Phi^{\prime}(0) \geq 0$ then according to Appendix A, $\Pi^{\varepsilon}(0) \geq \Pi(0)$ and consequently the local Vega index and the Vega index are both positive. Moreover, by using the integration by parts formula and setting $M_{T}^{S}:=\sup _{0 \leq s \leq T} S(s)$ for notational simplicity, we find that the above expectation equals:

$$
\frac{\sigma}{2} \int_{0}^{T / 2} E_{Q}\left[\Phi^{\prime}\left(M_{T}^{S}\right) M_{T}^{S} \mathbf{1}_{t \leq \tau}\right] d t-\frac{\sigma}{2} \int_{T / 2}^{T} E_{Q}\left[\Phi^{\prime}\left(M_{T}^{S}\right) M_{T}^{S} \mathbf{1}_{t \leq \tau}\right] d t .
$$

This expression is positive since the integrand $E_{Q}\left[\Phi^{\prime}\left(\sup _{0 \leq s \leq T} S(s)\right) \sup _{0 \leq s \leq T} S(s) \mathbf{1}_{t \leq \tau}\right]$ is a decreasing function as shown in the final part of the proof of proposition 3.3. Hence, this little toy example clearly shows that a perturbation of volatility close to maturity (take for instance $\sigma_{1}=0$ and $\sigma_{2}=1$ ) has less effect on the option prices than a similar perturbation at the beginning of the time interval $\left(\sigma_{1}=1\right.$ and $\left.\sigma_{2}=0\right)$. 


\section{Extensions on hedging of European options}

There are two aspects regarding the hedging problems that one may think of after the local change in volatility has been studied. One is how much more money is needed to put into the hedging strategy ${ }^{2}$ in order to cover for the change in volatility. This question is answered by analyzing the quantity $\left.\frac{\partial^{2} \Pi^{\varepsilon}}{\partial \varepsilon \partial S_{0}}(0)\right|_{\varepsilon=0}$, which of course can be carried out using similar calculations as the ones showed in the previous sections. The other question, though, is known as the goal problem and in our study it takes the form: given that we cannot or are not willing to add more money into the hedging portfolio what is the strategy to follow so that the chances of being able to cover the option are the highest? This question has been partly answered, although in a somewhat different setting, by Kulldorff (1993). Here, we briefly extend the results to time dependent volatility. The following analysis will be carried out under assumption A2 since the general case does not seem to lead to a tractable problem. We assume that the contingent claims to be studied are square integrable.

\subsection{The goal problem}

Let us start by giving a short resumé and a little extension of the goal problem. We refer to Karatzas (1996) for details and further references. Let us recall that the discounted value process of a self financing portfolio is given by the expression

$$
B(\cdot)^{-1} X^{x_{0}, \xi}(\cdot)=x_{0}+\int_{0} B(s)^{-1} \xi(s) \sigma(s) d V(s) \quad ; \quad \sigma(t):=\sigma+\varepsilon \hat{\sigma}(t) .
$$

Here $x_{0}$ is the initial wealth in our portfolio, i.e. $x_{0}=X^{x_{0}, \xi}(0)$, and the strategy $\xi(\cdot)$ represents the amount of money that is invested in the stock at each point in time. Of course we require $\xi(\cdot)$ to be an $\mathbb{F}$-adapted process. According to the extended Clark-Ocone formula any $\mathcal{F}_{T}$-measurable contingent claim $G^{\varepsilon} \in L^{2}(\Omega, \mathcal{F}, Q)$ can be expressed as

$$
B(T)^{-1} G^{\varepsilon}=B(T)^{-1} E_{Q}\left[G^{\varepsilon}\right]+\int_{0}^{T} B(s)^{-1} \bar{\pi}(s) \sigma(s) d V(s),
$$

where $\bar{\pi}(t)=\sigma(t)^{-1} B(t) B(T)^{-1} E_{Q}\left[D_{t} G^{\varepsilon} \mid \mathcal{F}_{t}\right]$ a.s. for all $t \in[0, T]$. Hence, starting with the initial wealth $u_{0}:=\Pi^{\varepsilon}(0)=B(T)^{-1} E_{Q}\left[G^{\varepsilon}\right]$ and using the strategy $\bar{\pi}(\cdot)$ we will at maturity obtain a perfect hedge, i.e. $X^{u_{0}, \bar{\pi}}(T)=G^{\varepsilon}$ almost surely. Moreover, in this case $B(\cdot)^{-1} X^{u_{0}, \bar{\pi}}(\cdot)$ is a $Q$-martingale and consequently the fair price of $G^{\varepsilon}$, i.e. the price consistent with no arbitrage opportunities, is given by $X^{u_{0}, \bar{\pi}}(t):=B(t) B(T)^{-1} E_{Q}\left[G^{\varepsilon} \mid \mathcal{F}_{t}\right]$.

Now, suppose that our initial wealth $x_{0}$ is less than the money required to obtain a perfect hedge, i.e. we assume $0<x_{0} \leq u_{0}$, then as we can no longer obtain a perfect hedge we will

\footnotetext{
${ }^{2}$ Note that when hedging a contingent claim, the number of units to be held in the underlying asset at each point in time, is given by the derivative of the option price with respect to the stock price.
} 
instead try to maximize the probability of a perfect hedge:

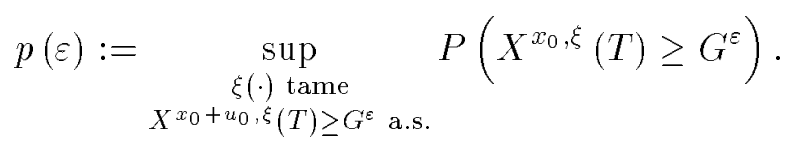

Note that in the case of a perfect hedge $X^{u_{0}, \bar{\pi}}(T)=G^{\varepsilon} \in L^{2}(\Omega, \mathcal{F}, Q)$, which implied that $B(\cdot)^{-1} X^{u_{0}, \bar{\pi}}(\cdot)$ was a $Q$-martingale. However, in this situation we do not have a terminal value for $X^{x_{0}, \xi}(\cdot)$ and therefore we have to impose the condition that $\xi(\cdot)$ is a tame strategy meaning that the process $\int_{0}^{\cdot} B(s)^{-1} \xi(s) \sigma(s) d V(s)$ is a.s. uniformly bounded from below by some real constant. By using Fatou's lemma we see that the discounted value process $B(\cdot)^{-1} X^{x_{0}, \xi}(\cdot)$ is a $Q$-supermartingale whenever the portfolio $\xi(\cdot)$ is tame, and in this case we also have that

$$
P\left(X^{x_{0}, \xi}(T) \geq G^{\varepsilon}\right)=P\left(B(T)^{-1} X^{x, \pi}(T) \geq 1\right),
$$

where $x=x_{0} / u_{0} \in[0,1]$ and $\pi(\cdot)=[\xi(\cdot)-\bar{\pi}(\cdot)] / u_{0}$, such that $\pi(\cdot)$ as well is a tame strategy. Moreover, it follows that the inequalities $X^{x_{0}+u_{0}, \xi}(T) \geq G^{\varepsilon}$ a.s. and $X^{x, \pi}(T) \geq 0$ a.s. are equivalent. Therefore the above stated problem is identical to solving

$$
G_{0}(x):=\sup _{\substack{\pi(\cdot) \text { tame } \\ X^{x, \pi}(T) \geq 0 \text { a.s. }}} P\left(B(T)^{-1} X^{x, \pi}(T) \geq 1\right) .
$$

Proposition 4.1 The maximal probability of obtaining a perfect hedge is given by

$$
p(\varepsilon)=N\left(N^{-1}\left(\frac{x_{0}}{u_{0}}\right)+|\alpha-r| \sqrt{\int_{0}^{T}[\sigma+\varepsilon \hat{\sigma}(t)]^{-2} d t}\right),
$$

with $u_{0}:=\Pi^{\varepsilon}(0)=e^{-r T} E_{Q}\left[G^{\varepsilon}\right]$. Moreover, the optimal strategy is given by the expression:

$$
\xi^{*}(t)=\bar{\pi}(t)+u_{0} \pi^{*}(t)
$$

where

$$
\bar{\pi}(t)=\sigma(t)^{-1} B(t) B(T)^{-1} E_{Q}\left[D_{t} G^{\varepsilon} \mid \mathcal{F}_{t}\right]
$$

and

$$
\begin{aligned}
\pi^{*}(t) & =e^{r t} \varphi\left(\frac{\int_{0}^{t} \sigma(s)^{-1} d V(s)+N^{-1}\left(\frac{x_{0}}{u_{0}}\right) \sqrt{\int_{0}^{T} \sigma(s)^{-2} d s}}{\sqrt{\int_{t}^{T} \sigma(s)^{-2} d s}}\right) \frac{\sigma(t)^{-2}}{\sqrt{\int_{t}^{T} \sigma(s)^{-2} d s}} \\
& =e^{r t} \varphi\left(N^{-1}\left(B(T)^{-1} X^{x, \hat{\pi}}(t)\right)\right) \frac{\sigma(t)^{-2}}{\sqrt{\int_{t}^{T} \sigma(s)^{-2} d s}} .
\end{aligned}
$$

Here, we have used the notation $\sigma(t):=\sigma+\varepsilon \hat{\sigma}(t)$, and let $\varphi(\cdot)$ denote the derivative of $N(\cdot)$. 
It is interesting to see that the expressions for the optimal strategies $\left(\pi^{*}, \xi^{*}\right)$ do not depend on the stock appreciation rate $\alpha$. This, however, ceases to be the case whenever the risk premium $|\alpha-r|$ is time dependent. Note that although the strategy $\bar{\pi}(\cdot)$ satisfies the requirements in the original problem, it is never optimal.

Proof. In order to solve problem (4.2), we will start by showing that $G_{0}(x)=G(x)=G_{*}(x)$ for $x \in[0,1]$, where

$$
\begin{aligned}
G(x) & :=\sup _{\pi(\cdot) \in \mathcal{H}(x)} P\left(B(T)^{-1} X^{x, \pi}(T)=1\right) \\
G_{*}(x) & :=\sup _{B \in \mathcal{F}_{T}, Q(B) \leq x} P(B)
\end{aligned}
$$

and

$$
\mathcal{H}(x)=\left\{\pi:[0, T] \times \Omega \rightarrow \mathbb{R} \mid \int_{0}^{T} \pi^{2}(t) d t<\infty, \text { and } 0 \leq B(t)^{-1} X^{x, \pi}(t) \leq 1 \text { a.s. for all } t\right\} .
$$

We get immediately that $G(x) \leq G_{0}(x)$ and $G(x) \leq G_{*}(x)$. Moreover, for any $\pi(\cdot)$ tame with $X^{x, \pi}(T) \geq 0$ a.s., we have

$$
\begin{aligned}
Q\left(B(T)^{-1} X^{x, \pi}(T) \geq 1\right) & =E_{Q}\left[1\left\{B(T)^{-1} X^{x, \pi}(T) \geq 1\right\}\right] \\
& \leq E_{Q}\left[B(T)^{-1} X^{x, \pi}(T)\right] \\
& \leq x
\end{aligned}
$$

since the discounted value process is a $Q$-supermartingale for $\pi(\cdot)$ tame, which gives $G_{0}(x) \leq$ $G_{*}(x)$. Summing up we have $G(x) \leq G_{0}(x) \leq G_{*}(x)$. We therefore only have to establish the equality $G_{*}(x)=G(x)$. However, in order to do this we will first start by characterizing $G_{*}(x)$. According to the Newman-Pearson lemma it follows that if we can find a number $k=k(x)>0$ for which the event

$$
B_{k}:=\left\{\frac{d P}{d Q} \geq k\right\} \quad \text { has } \quad Q\left(B_{k}\right)=x,
$$

then $G_{*}(x)=P\left(B_{k}\right)$. We define the risk premium $\theta(t)=[\alpha-r] / \sigma(t)$, such that

$$
\frac{d P}{d Q}=\exp \left(\int_{0}^{T} \theta(t) d W(t)+\frac{1}{2} \int_{0}^{T} \theta(t)^{2} d t\right)=\exp \left(\int_{0}^{T} \theta(t) d V(t)-\frac{1}{2} \int_{0}^{T} \theta(t)^{2} d t\right),
$$

and

$$
Q\left(B_{k}\right)=N\left(-\frac{\frac{1}{2} \int_{0}^{T} \theta(t)^{2} d t+\ln k}{\sqrt{\int_{0}^{T} \theta(t)^{2} d t}}\right)
$$


where as usual $N(\cdot)$ denotes the cumulative distribution function of a standard normal random variable. Setting $Q\left(B_{k}\right)=x$, we get that

$$
\ln k=-N^{-1}(x) \sqrt{\int_{0}^{T} \theta(t)^{2} d t}-\frac{1}{2} \int_{0}^{T} \theta(t)^{2} d t
$$

and consequently

$$
G_{*}(x)=P\left(B_{k}\right)=N\left(N^{-1}(x)+\sqrt{\int_{0}^{T} \theta(t)^{2} d t}\right) .
$$

Now, we try to find a portfolio $\pi^{*}(\cdot) \in \mathcal{H}(x)$ such that $\left\{B(T)^{-1} X^{x, \pi^{*}}(T)=1\right\}=B_{k}$ a.s., since it then follows that $G(x)=G_{*}(x)$. We define $\hat{X}(\cdot)=E_{Q}\left[1\left\{B_{k}\right\} \mid \mathcal{F}.\right]=Q\left(B_{k} \mid \mathcal{F}\right.$. $)$. Hence, $\hat{X}(\cdot)$ is a $Q$-martingale taking values in $[0,1]$, with $\hat{X}(0)=Q\left(B_{k}\right)=x$ and $\hat{X}(T)=$ $E_{Q}\left[1\left\{B_{k}\right\} \mid \mathcal{F}_{T}\right]=1\left\{B_{k}\right\}$. From the Clark-Ocone formula and the martingale property we have the representation $\hat{X}(\cdot)=x+\int_{0}^{\cdot} E_{Q}\left[D_{s} \hat{X}(T) \mid \mathcal{F}_{s}\right] d V(s)$, and the integrability condition $\int_{0}^{T} E_{Q}\left[\left(E_{Q}\left[D_{s} \hat{X}(T) \mid \mathcal{F}_{s}\right]\right)^{2}\right] d s<+\infty$. Now, defining

$$
\pi^{*}(\cdot)=B(\cdot) E_{Q}[D . \hat{X}(T) \mid \mathcal{F} .] \sigma(\cdot)^{-1},
$$

we have according to $(4.1)$ that $B(\cdot)^{-1} X^{x, \pi^{*}}(\cdot)=\hat{X}(\cdot)$ and consequently $\pi^{*}(\cdot) \in \mathcal{H}(x)$. This also shows that $G(x)=G_{*}(x)$ concluding the first part of the proof.

In order to obtain a representation for the optimal portfolio we interchange the order of Malliavin derivation and conditional expectation, see Bermin (1998b), and use the martingale property of $\hat{X}(\cdot)$

$$
E_{Q}\left[D_{s} \hat{X}(T) \mid \mathcal{F}_{s}\right]=D_{s} E_{Q}\left[\hat{X}(T) \mid \mathcal{F}_{s}\right]=D_{s} \hat{X}(s)=D_{s} Q\left(B_{k} \mid \mathcal{F}_{s}\right)
$$

The conditional probability $Q\left(B_{k} \mid \mathcal{F}_{s}\right)$ can be calculated explicitly:

$$
Q\left(B_{k} \mid \mathcal{F}_{s}\right)=N\left(\frac{\int_{0}^{s} \theta(t) d V(t)-\frac{1}{2} \int_{0}^{T} \theta(t)^{2} d t-\ln k}{\sqrt{\int_{s}^{T} \theta(t)^{2} d t}}\right) .
$$

Finally, by using the chain rule and the relation $D_{s} \int_{0}^{s} \theta(t) d V(t)=\theta(s)$, we get that

$$
E_{Q}\left[D_{s} \hat{X}(T) \mid \mathcal{F}_{s}\right]=\varphi\left(\frac{\int_{0}^{s} \theta(t) d V(t)+N^{-1}(x) \sqrt{\int_{0}^{T} \theta(t)^{2} d t}}{\sqrt{\int_{s}^{T} \theta(t)^{2} d t}}\right) \frac{\theta(s)}{\sqrt{\int_{s}^{T} \theta(t)^{2} d t}},
$$

where we have used the optimal expression for $\ln k$, i.e. equation (4.3), and introduced the function $\varphi(\cdot)=\frac{d N}{d x}(\cdot)$. In fact we can express $\pi^{*}(\cdot)$ by a feed-back representation. In order to 
see this, note that

$$
B(s)^{-1} X^{x, \pi^{*}}(s)=Q\left(B_{k} \mid \mathcal{F}_{s}\right)=N\left(\frac{\int_{0}^{s} \sigma(t)^{-1} d V(t)+N^{-1}(x) \sqrt{\int_{0}^{T} \sigma(t)^{-2} d t}}{\sqrt{\int_{s}^{T} \sigma(t)^{-2} d t}}\right) .
$$

Hence

$$
\int_{0}^{s} \sigma(t)^{-1} d V(t)=N^{-1}\left(B(s)^{-1} X^{x, \pi^{*}}(s)\right) \sqrt{\int_{s}^{T} \sigma(t)^{-2} d t}-N^{-1}(x) \sqrt{\int_{0}^{T} \sigma(t)^{-2} d t}
$$

from which the proof follows.

One undesirable feature of the optimization problem considered in this proposition is that the set of admissible strategies cannot be chosen arbitrarily, i.e. we have to consider strategies such that $\xi(\cdot)$ is tame and $X^{x_{0}+u_{0}, \xi}(T) \geq G^{\varepsilon}$ almost surely. Without this restriction one could not obtain the sequence of equivalences between the optimal problems stated at the beginning of the proof. To some extent thus, the problem is somewhat artificial since the class of strategies for which $X^{x_{0}+u_{0}, \xi}(T) \geq G$ a.s. is indeed very large. Furthermore, there is not a completely obvious economic interpretation for why we should seek the optimal strategy within this class. Nevertheless, we find that the local Vega index is an important quantity in determining how fast perfect hedging is achieved. This is the topic of the next subsection.

\subsection{The speed of convergence}

We consider the situation were the time 0 price of a contingent claim $G$ in the classical BlackScholes model is given by the quantity $x_{0}:=\Pi(0)=e^{-r T} E_{Q}[G]$, whereas in the perturbed model the corresponding contingent claim is worth the amount $u_{0}:=\Pi^{\varepsilon}(0)=e^{-r T} E_{Q}\left[G^{\varepsilon}\right]$. Now, let us suppose that we cannot or are not willing to put more money into the hedging strategy than $x_{0}$ where it is assumed that $x_{0} \leq u_{0}$. Alternatively, suppose that we don't know how to explicitly calculate the quantity $u_{0}$ although we have managed to show the inequality $x_{0} \leq u_{0}$, see for instance Appendix A. In both situations though, we can no longer obtain a perfect hedge and therefore we will instead try to maximize the probability of a perfect hedge as in proposition 4.1:

$$
p(\varepsilon)=N\left(N^{-1}\left(\frac{\Pi(0)}{\Pi^{\varepsilon}(0)}\right)+|\alpha-r| \sqrt{\int_{0}^{T}[\sigma+\varepsilon \hat{\sigma}(t)]^{-2} d t}\right) .
$$

Now, letting $\varepsilon \rightarrow 0$ we see that $p(\varepsilon) \rightarrow 1$ according to the assumption $\sigma+\varepsilon \hat{\sigma}(t) \geq \sigma_{\min }$ for all $\varepsilon$ and $t \in\left[0, T^{*}\right]$. Hence, in the limit we will obtain a perfect hedge. So, the question is: how fast will $p(\varepsilon)$ converges to one? As we will see, the quantity that determines the speed is the ratio $\left.\frac{\partial \Pi^{\varepsilon}}{\partial \varepsilon}(0)\right|_{\varepsilon=0} / \Pi(0)$. This gives another motivation for the importance of studying the local Vega index. Throughout this subsection we will make the following assumption: 
Assumption The perturbed price $\Pi^{\varepsilon}(0)$ has a Taylor expansion of order 2 around $\varepsilon=0$, in the sense that

$$
\Pi^{\varepsilon}(0)=\Pi(0)+\left.\frac{\partial \Pi^{\varepsilon}}{\partial \varepsilon}(0)\right|_{\varepsilon=0} \varepsilon+G(\varepsilon) \varepsilon^{2},
$$

where $G(\cdot)$ is differentiable around 0 , and $|G(\varepsilon)| \leq C_{1}$ for $\varepsilon \leq \bar{\varepsilon}$.

By using the same techniques as in the previous sections where we have computed the local Vega indices for different options it can be proved that these prices satisfy the above assumption.

Proposition 4.2 The maximal probability of obtaining a perfect hedge in (4.4) has the property

$$
\lim _{\varepsilon \rightarrow 0} \frac{1-p(\varepsilon)}{\varepsilon \exp \left(-c N^{-1}(1-\varepsilon)\right)}=\left.\exp \left(-c^{2} / 2\right) \frac{\partial \Pi^{\varepsilon}}{\partial \varepsilon}(0)\right|_{\varepsilon=0} / \Pi(0),
$$

where $c=\frac{|\alpha-r|}{\sigma} \sqrt{T}$.

Note that $N^{-1}(1-\varepsilon) \approx \sqrt{-\ln \varepsilon}$, hence $\exp \left(-c N^{-1}(1-\varepsilon)\right)$ goes to zero slower than any polynomial.

Proof. We start by expanding the perturbed price $\Pi^{\varepsilon}(0)$ around $\varepsilon=0$. Consequently, $\frac{\Pi(0)}{\Pi^{\varepsilon}(0)}=1-\frac{\Pi^{\varepsilon}(0)-\Pi(0)}{\Pi^{\varepsilon}(0)}$ is given by

$$
1-\frac{\left.\frac{\partial \Pi^{\varepsilon}}{\partial \varepsilon}(0)\right|_{\varepsilon=0} \varepsilon+G(\varepsilon) \varepsilon^{2}}{\Pi(0)+\left.\frac{\partial \Pi^{\varepsilon}}{\partial \varepsilon}(0)\right|_{\varepsilon=0} \varepsilon+G(\varepsilon) \varepsilon^{2}}=1-\frac{\left.\frac{\partial \Pi^{\varepsilon}}{\partial \varepsilon}(0)\right|_{\varepsilon=0}}{\Pi(0)} \varepsilon+F(\varepsilon) \varepsilon^{2},
$$

where we now have that $|F(\varepsilon)| \leq C_{2}$ for $\varepsilon \leq \bar{\varepsilon}$. In order to simplify the notations we define $a:=\left.\frac{\partial \Pi^{\varepsilon}}{\partial \varepsilon}(0)\right|_{\varepsilon=0} / \Pi(0)$ and $c_{\varepsilon}:=|\alpha-r| \sqrt{\int_{0}^{T}[\sigma+\varepsilon \hat{\sigma}(t)]^{-2} d t}$, with $c:=\lim _{\varepsilon \rightarrow 0} c_{\varepsilon}=\frac{|\alpha-r|}{\sigma} \sqrt{T}$. Let $f(\varepsilon):=1-p(\varepsilon)$ such that $f(0)=0$ and

$$
\begin{aligned}
\frac{d f}{d \varepsilon}(\varepsilon)= & \frac{\varphi\left(N^{-1}\left(1-a \varepsilon+F(\varepsilon) \varepsilon^{2}\right)+c_{\varepsilon}\right)}{\varphi\left(N^{-1}\left(1-a \varepsilon+F(\varepsilon) \varepsilon^{2}\right)\right)}\left(a-2 \varepsilon F(\varepsilon)-\frac{d F}{d \varepsilon}(\varepsilon) \varepsilon^{2}\right) \\
& -\varphi\left(N^{-1}\left(1-a \varepsilon+F(\varepsilon) \varepsilon^{2}\right)+c_{\varepsilon}\right) \frac{d c_{\varepsilon}}{d \varepsilon} .
\end{aligned}
$$

Note that the last expression follows from the identity $\frac{\Pi(0)}{\Pi^{\varepsilon}(0)}=1-a \varepsilon+F(\varepsilon) \varepsilon^{2}$, and the derivation rule for inverse functions. Now, we set $L=\lim _{\varepsilon \rightarrow 0} \frac{f(\varepsilon)}{\varepsilon \exp \left(-c N^{-1}(1-\varepsilon)\right)}$ and according to L'Hopital's rule we find that

$$
L=\lim _{\varepsilon \rightarrow 0} \frac{\frac{d f}{d \varepsilon}(\varepsilon)}{\exp \left(-c N^{-1}(1-\varepsilon)\right)\left(1+\varepsilon c[\varphi(1-\varepsilon)]^{-1}\right)} .
$$

Without too much problem we see that $\lim _{\varepsilon \rightarrow 0} \varepsilon c[\varphi(1-\varepsilon)]^{-1}=0$, hence we conclude that

$$
L=\lim _{\varepsilon \rightarrow 0} \frac{\frac{d f}{d \varepsilon}(\varepsilon)}{\exp \left(-c N^{-1}(1-\varepsilon)\right)} .
$$


The derivative $\frac{d f}{d \varepsilon}(\varepsilon)$ consists of two terms where the second term will actually not contribute. Hence, after several applications of L'Hopital's rule and somewhat messy algebra we find that

$$
\lim _{\varepsilon \rightarrow 0} \frac{\varphi\left(N^{-1}\left(1-a \varepsilon+F(\varepsilon) \varepsilon^{2}\right)+c_{\varepsilon}\right) \frac{d c_{\varepsilon}}{d \varepsilon}}{\exp \left(-c N^{-1}(1-\varepsilon)\right)}=0,
$$

leaving us with the expression

$$
L=\lim _{\varepsilon \rightarrow 0} \frac{\varphi\left(N^{-1}\left(1-a \varepsilon+F(\varepsilon) \varepsilon^{2}\right)+c_{\varepsilon}\right)\left(a-2 \varepsilon F(\varepsilon)-\frac{d F}{d \varepsilon}(\varepsilon) \varepsilon^{2}\right)}{\varphi\left(N^{-1}\left(1-a \varepsilon+F(\varepsilon) \varepsilon^{2}\right)\right) \exp \left(-c N^{-1}(1-\varepsilon)\right)} .
$$

To evaluate this expression we will introduce some auxiliary variables. Define

$$
\begin{aligned}
M & =\lim _{\varepsilon \rightarrow 0} \frac{\exp \left(-c_{\varepsilon} N^{-1}\left(1-a \varepsilon+F(\varepsilon) \varepsilon^{2}\right)\right)}{\exp \left(-c N^{-1}(1-\varepsilon)\right)}, \\
M_{1} & =\lim _{\varepsilon \rightarrow 0} \frac{\exp \left(-N^{-1}\left(1-a \varepsilon+F(\varepsilon) \varepsilon^{2}\right)\right)}{\exp \left(-N^{-1}(1-\varepsilon)\right)} .
\end{aligned}
$$

Now as $\varphi(x+y) / \varphi(x)=\exp \left(-y^{2} / 2\right) \exp (-x y)$, we get that

$$
L:=M \lim _{\varepsilon \rightarrow 0} \exp \left(-c_{\varepsilon}^{2} / 2\right) \lim _{\varepsilon \rightarrow 0}\left(a-2 \varepsilon F(\varepsilon)-\frac{d F}{d \varepsilon}(\varepsilon) \varepsilon^{2}\right)=M \exp \left(-c^{2} / 2\right) a .
$$

We will evaluate $M$ in a sequence of steps. However, first we notice that we can rewrite

$$
M:=M_{1}^{c} \lim _{\varepsilon \rightarrow 0} \exp \left(\left(c-c_{\varepsilon}\right) N^{-1}\left(1-a \varepsilon+F(\varepsilon) \varepsilon^{2}\right)\right)=M_{1}^{c} .
$$

To verify that $\left(c-c_{\varepsilon}\right) N^{-1}\left(1-a \varepsilon+F(\varepsilon) \varepsilon^{2}\right) \rightarrow 0$, note that for $N^{-1}\left(1-a \varepsilon+F(\varepsilon) \varepsilon^{2}\right) \geq \alpha^{-1}$ we have

$$
\text { (4.6) } a \varepsilon-F(\varepsilon) \varepsilon^{2}:=\int_{N^{-1}\left(1-a \varepsilon+F(\varepsilon) \varepsilon^{2}\right)}^{\infty} \varphi(y) d y \leq \frac{\alpha}{\sqrt{2 \pi}} \exp \left(-\frac{1}{2}\left[N^{-1}\left(1-a \varepsilon+F(\varepsilon) \varepsilon^{2}\right)\right]^{2}\right) \text {. }
$$

Hence, $N^{-1}\left(1-a \varepsilon+F(\varepsilon) \varepsilon^{2}\right)$ is of order $\sqrt{-\ln \varepsilon}$ for $\varepsilon$ small. However, $\left(c-c_{\varepsilon}\right)$ is of order $\varepsilon$ and (4.5) follows. To evaluate $M_{1}=\lim _{\varepsilon \rightarrow 0} \exp \left(-\left[N^{-1}\left(1-a \varepsilon+F(\varepsilon) \varepsilon^{2}\right)-N^{-1}(1-\varepsilon)\right]\right)$ we start by using the mean value theorem

$$
\begin{aligned}
\varepsilon-a \varepsilon+F(\varepsilon) \varepsilon^{2} & =\int_{N^{-1}(1-\varepsilon)}^{N^{-1}\left(1-a \varepsilon+F(\varepsilon) \varepsilon^{2}\right)} \varphi(y) d y \\
& =\frac{1}{\sqrt{2 \pi}} \exp \left(-y^{2}(\varepsilon) / 2\right)\left[N^{-1}\left(1-a \varepsilon+F(\varepsilon) \varepsilon^{2}\right)-N^{-1}(1-\varepsilon)\right],
\end{aligned}
$$

for some $y(\varepsilon) \in\left[N^{-1}(1-\varepsilon), N^{-1}\left(1-a \varepsilon+F(\varepsilon) \varepsilon^{2}\right)\right]$. Intuitively, $M_{1}$ should be equal to one and this is what we want to show. It is sufficient though to show that $N^{-1}\left(1-a \varepsilon+F(\varepsilon) \varepsilon^{2}\right)-$ $N^{-1}(1-\varepsilon) \rightarrow 0$ or rather that $\varepsilon \exp \left(y^{2}(\varepsilon) / 2\right) \rightarrow 0$. Hence, we are left to verify:

$$
\lim _{\varepsilon \rightarrow 0} \varepsilon \exp \left(\left[N^{-1}(1-\varepsilon) \vee N^{-1}\left(1-a \varepsilon+F(\varepsilon) \varepsilon^{2}\right)\right]^{2} / 2\right)=0 .
$$


First we assume that $N^{-1}\left(1-a \varepsilon+F(\varepsilon) \varepsilon^{2}\right)>N^{-1}(1-\varepsilon)$. From (4.6) it then follows that for $N^{-1}\left(1-a \varepsilon+F(\varepsilon) \varepsilon^{2}\right) \geq \alpha^{-1}$, and some constant $C>1$, we have that

$$
\begin{aligned}
\lim _{\varepsilon \rightarrow 0} \varepsilon \exp \left(\left[N^{-1}\left(1-a \varepsilon+F(\varepsilon) \varepsilon^{2}\right)\right]^{2} / 2\right) & \leq \lim _{\varepsilon \rightarrow 0} \frac{C / \sqrt{2 \pi}}{N^{-1}\left(1-a \varepsilon+F(\varepsilon) \varepsilon^{2}\right)} \lim _{\varepsilon \rightarrow 0} \frac{1}{(a-F(\varepsilon) \varepsilon)} \\
& =0 .
\end{aligned}
$$

The other case, i.e. $N^{-1}(1-\varepsilon) \geq N^{-1}\left(1-a \varepsilon+F(\varepsilon) \varepsilon^{2}\right)$, can be treated similarly which proves (4.7). Therefore, $M_{1}=M=1$ and $L=\exp \left(-c^{2} / 2\right) a$.

\section{$5 \quad$ Practical use and summary}

In this section, let us assume A2 in order to simplify the discussion. At a first sight, the implementation of the local Vega index $\left.\frac{\partial \Pi^{\varepsilon}}{\partial \varepsilon}(0)\right|_{\varepsilon=0}$ might seem a little strange due to the fact that the local volatility, i.e. $\varepsilon \hat{\sigma}(\cdot)$, is over specified. Hence, suppose that the manager believes that the future volatility over the time period $[0, T]$ will be given by the deterministic function $\sigma(\cdot)$. He then solves backwards for $\varepsilon \hat{\sigma}(\cdot)=\sigma(\cdot)-\sigma$. Now, depending on what value of $\varepsilon$ that is fixed at the beginning the manager will obtain different functions $\hat{\sigma}(\cdot)$. To be precise we see that the function $\hat{\sigma}(\cdot)$ is uniquely defined up to a multiplicative constant. This is of course a problem as we found that for all the options studied in this paper we had the relationship

$$
\left.\frac{\partial \Pi^{\varepsilon}}{\partial \varepsilon}(0)\right|_{\varepsilon=0}=\int_{0}^{T} \bar{\mu}(s, T) \hat{\sigma}(s) d s \frac{\partial \Pi}{\partial \sigma}(0),
$$

where $\bar{\mu}(\cdot, T)$ was a density function independent of $\varepsilon$ and $\hat{\sigma}(\cdot)$. Therefore the local Vega index $\left.\frac{\partial \Pi^{\varepsilon}}{\partial \varepsilon}(0)\right|_{\varepsilon=0}$ is unique up to a multiplicative constant as well. It follows that the quantity of interest for practical situations is $\left.\varepsilon \frac{\partial \Pi^{\varepsilon}}{\partial \varepsilon}(0)\right|_{\varepsilon=0}$ and not the local Vega index by itself. Hence, the local Vega neutrality approach used by a financial manager should actually be written as

$$
\left.\sum_{i} \varepsilon \frac{\partial \Pi_{i}^{\varepsilon}}{\partial \varepsilon}(0)\right|_{\varepsilon=0}:=\sum_{i} \frac{\partial \Pi_{i}}{\partial \sigma}(0)\left(\int_{0}^{T_{i}} \bar{\mu}_{i}\left(s, T_{i}\right) \sigma(s) d s-\sigma\right) \approx 0,
$$

where the sum, as before, is taken over every contingent claim traded by the company. Note that for a portfolio composed of simple contingent claims with a fixed maturity $T$, one has

$\bar{\mu}_{i}(\cdot, T)=\frac{1}{T}$ and the local Vega neutrality concept corresponds exactly to the classical Vega neutrality approach $\sum_{i} \frac{\partial \Pi_{i}}{\partial \sigma}(0) \approx 0$. However, for all other cases these two criteria do not coincide.

In order to study the price variations we use the integral formula:

$$
\Pi^{1}(0)-\Pi^{0}(0)=\left.\int_{0}^{1} \frac{\partial \Pi^{\varepsilon}}{\partial \varepsilon}(0) d \varepsilon \approx \frac{\partial \Pi^{\varepsilon}}{\partial \varepsilon}(0)\right|_{\varepsilon=0},
$$


where the price $\Pi^{1}(0)$ corresponds to the case where the stock has the volatility $\sigma+\hat{\sigma}(\cdot)$. This analysis can of course also be related to the classical Vega index in the sense that

$$
\Pi_{\sigma_{1}}^{0}(0)-\Pi^{0}(0)=\int_{\sigma}^{\sigma_{1}} \frac{\partial \Pi_{v}^{0}}{\partial \sigma}(0) d v \approx \frac{\partial \Pi_{\sigma}^{0}}{\partial \sigma}(0) \cdot\left(\sigma_{1}-\sigma\right)
$$

where we let $\Pi_{\sigma_{1}}^{0}(\cdot)$ denote the price given that the volatility is constant and equal to $\sigma_{1}$. Note that these are just first order approximations. By including higher order terms more accuracy can be gained, however this would of course be more complicated. Another possibility is to use Riemann sum approximations.

In order to show the basic idea we take the first order approximations above, and estimate the yield volatility, i.e. the constant volatility $\sigma_{1}$ that makes $\Pi^{1}(0) \approx \Pi_{\sigma_{1}}^{0}(0)$, by setting $\sigma_{1}=\int_{0}^{T} \bar{\mu}(s, T) \sigma(s) d s$. This may be of interest since there exists no closed form solution for the price of most exotic options whenever the volatility is time dependent. In order to see how the method works, we consider the following example:

Example 5.1 Let us again consider the standard call option with payoff $G=\max (S(T)-K, 0)$ for some constant strike price $K$, and assume that the dynamics of the stock price are given by

$$
d S(t)=r S(t) d t+\sigma(t) S(t) d V(t),
$$

for some strictly positive deterministic function $\sigma(\cdot)$ in $L^{2}([0, T])$. By using the results in example 1.1, the time 0 price of the option is given by

$$
\Pi(0)=S_{0} N\left(d_{1}\right)-e^{-r T} K N\left(d_{1}-\sqrt{\Sigma}\right),
$$

and $d_{1}$ is defined by

$$
d_{1}=\frac{\ln \left(S_{0} / K\right)+r T+\frac{1}{2} \Sigma}{\sqrt{\Sigma}} \quad ; \quad \Sigma=\int_{0}^{T} \sigma(t)^{2} d t .
$$

The approximate option price, denoted $\tilde{\Pi}(0)$, is given by the Black-Scholes formula evaluated at the yield volatility $\sigma_{1}=\frac{1}{T} \int_{0}^{T} \sigma(s) d s$, that is

$$
\tilde{\Pi}(0)=S_{0} N\left(\tilde{d}_{1}\right)-e^{-r T} K N\left(\tilde{d}_{1}-\sigma_{1} \sqrt{T}\right) \quad ; \quad d_{1}=\frac{\ln \left(S_{0} / K\right)+r T+\frac{1}{2} \sigma_{1}^{2} T}{\sigma_{1} \sqrt{T}} .
$$

However, since $\sigma_{1}^{2} T=\frac{1}{T}\left(\int_{0}^{T} \sigma(s) d s\right)^{2} \leq \int_{0}^{T} \sigma(s)^{2} d s$, it follows from El Karoui et al. (1998) that $\tilde{\Pi}(0) \leq \Pi(0)$, where we have equality if and only if $\sigma(\cdot)$ is constant. Hence, to improve the approximation it may be necessary to use a quadratic Taylor expansion for the classical Vega index and Riemann sums for the local Vega index. It would be interesting to use these ideas to price path-dependent options.

To sum up, we have in this paper addressed a problem arising naturally in risk management: how can a financial manager protect the company's position once the manager has a personal 
belief about the future development of the market. Typical questions that can be studied are for instance what happens to the option prices if the next two weeks will be a very unstable period, or what happens to the option prices if the volatility will drop in a month as predicted by a time series analysis.

The local Vega index introduced here should be helpful to answer these and other related questions. For example, what is the relationship between this index and presence of asymmetric information and particularly existence of inside information. Another interesting question is: what is the amount of rebalancing needed in order to keep a portfolio stable with respect to the local Vega index? We believe that this amount is smaller than the amount needed when using the classical Vega neutrality concept since the local Vega index incorporates anticipated time dependent volatility structures. We leave it as open questions to see if our results might be useful in order to detect such phenomena.

The local Vega index is a natural extension of the classical Vega index, i.e. the price derivative with respect to the constant volatility, in the sense that we perturb the volatility in different directions. For all the contingent claims studied in this paper, we show that the local Vega index can be expressed as a weighted average of the perturbation in volatility. In the case that one assumes that the volatility and the rate of interest are constant and the perturbation in volatility only depends on time then this average is multiplied by the classical Vega index, giving a clear relationship between the classical Vega index and the local one defined here. Moreover, in the case of path-dependent options these weighted averages have in general the property of putting less and less weight to events in the future. Hence, a financial manager should according to this result think in short terms and do not worry (that much) about the future.

As an application of the results we show how to derive approximate prices of options for which no closed form solution exists in the case where the volatility is time dependent. This is done by means of a constant yield volatility under the assumption that a closed form solution indeed exists whenever the underlying security has a constant volatility.

We also study the well-known goal problem of maximizing the probability of a perfect hedge and show that the speed of convergence depends on the local Vega index.

\section{References}

Bermin, H.-P. (1998a) Essays on Lookback and Barrier Options: A Malliavin Calculus Approach, Ph.D.-thesis, Department of Economics, Lund University.

Bermin, H.-P. (1998b) Hedging options: the Malliavin calculus approach versus the $\Delta$-hedging approach, Working paper, Department of Economics, Lund University.

Dupire, B (1994) Pricing with a smile, Risk, 7, 18-20. 
El Karoui, N., Jeanblanc-Picqué, M., and Shreve, S.E. (1998) On the robustness of the BlackScholes equation, Mathematical Finance, 8, 93-126.

Fournié, E., Lasry, J.-M., Lebuchoux, J., Lions, P.-L. and Touzi, N. (1999) An application of Malliavin calculus to Monte Carlo methods in finance, Finance and Stochastics, 4 , to be published.

Harrison, J.M. and Pliska, S.R. (1981) Martingales and stochastic integrals in the theory of continuous trading, Stochastic Processes and Their Applications, 11, 215-260.

Hobson, D. (1997) Robust hedging via coupling, Working paper, School of Mathematical Sciences, University of Bath.

Karatzas, I. (1996) Lectures on the Mathematics of Finance, CRM Monographs 8, American Mathematical Society.

Karatzas, I. and Ocone, D. (1991) A generalized Clark representation formula, with applications to optimal portfolios. Stochastics and Stochastic Reports, 34, 187-220.

Karatzas, I. and Shreve, S.E. (1988) Brownian Motion and Stochastic Calculus, second edition, Springer-Verlag.

Kulldorff, M. (1993) Optimal control of favorable games with a time- limit, SIAM Journal of Control \&3 Optimization, 31, 52-69.

Kusuoka, S. and Stroock, D.W. (1984) Application of the Malliavin calculus I, In: Stochastic Analysis, Proceedings of the Taniguchi International Symposium on Stochastic Analysis, Katata and Kyoto, 1982, ed.: Itô, K., Kinokuniya/North-Holland, Tokyo, 271-306.

Nualart, D. (1995) The Malliavin Calculus and Related Topics, Springer-Verlag.

Peskir, G. (1998) Optimal stopping of the maximum process: the maximality principle, Annals of Probability, 26, 1614-1640.

Protter, P. (1990) Stochastic Integration and Differential Equations: a unified approach, SpringerVerlag.

Rudin, W. (1976) Principles of Mathematical Analysis: third edition, McGraw-Hill.

Seshadri, V. (1988) Exponential models, Brownian motion, and independence, Canadian Journal of Statistics, 16, 209-221.

Taniguchi, S. (1985) Applications of Malliavin's calculus to time-dependent systems of heat equations, Osaka Journal of Mathematics, 22, 307-320.

Üstünel, A.S. (1995) An Introduction to Analysis on Wiener Space, Springer Lecture Notes in Mathematics 1610. 


\section{Appendix A}

Theorem A.1 Assume A2 and that the local volatility perturbation $\hat{\sigma}(\cdot) \geq 0$. Then for Asian options we have that $\Pi^{\varepsilon}(\cdot) \geq \Pi(\cdot)$ for all $\varepsilon$. Furthermore, if we assume that the payoff function $\Phi^{\prime}(0) \geq 0$ then for lookback options we have that $\Pi^{\varepsilon}(\cdot) \geq \Pi(\cdot)$ for all $\varepsilon$.

Proof. Let us introduce the stochastic processes (state variables) $Y_{1}^{\varepsilon}(\cdot)=\int_{0} w(u) S^{\varepsilon}(u) d u$ and $Y_{2}^{\varepsilon}(\cdot)=\sup _{0 \leq u \leq .} S^{\varepsilon}(u)$. Thanks to the Markov property, the price of a Asian or a Lookback option can always be written in the form $\Pi_{i}^{\varepsilon}(t)=\pi_{i}^{\varepsilon}\left(t, S^{\varepsilon}(t), Y_{i}^{\varepsilon}(t)\right)$, where the deterministic function $\pi_{i}^{\varepsilon}(\cdot)$ is defined by

$$
\pi_{i}^{\varepsilon}(t, x, y)=e^{-r(T-t)} E_{Q}\left[G_{i}^{\varepsilon} \mid S^{\varepsilon}(t)=x, Y_{i}^{\varepsilon}(t)=y\right] \quad ; \quad i=1,2 .
$$

Here $G_{i}^{\varepsilon}$ denotes the payoff at maturity of the different contingent claims, i.e. $G_{i}^{\varepsilon}=\Phi\left(Y_{i}^{\varepsilon}(T)\right)$. The deterministic function $\pi_{i}^{\varepsilon}(\cdot)$ satisfies the following boundary problem:

$$
\begin{aligned}
-\frac{\partial \pi_{i}^{\varepsilon}}{\partial t}(t, x, y)+r \pi_{i}^{\varepsilon}(t, x, y) & =\mathcal{A}_{i, t}^{\varepsilon} \pi_{i}^{\varepsilon}(t, x, y) \\
1\{i=2\} \frac{\partial \pi_{i}^{\varepsilon}}{\partial y}(t, x, x) & =0 \\
\pi_{i}^{\varepsilon}(T, x, y) & =\Phi(y),
\end{aligned}
$$

where $\mathcal{A}_{i, t}^{\varepsilon}$ denotes the infinitesimal generator of the pair $\left(S^{\varepsilon}(t), Y_{i}^{\varepsilon}(t)\right)$. It can be shown that in our case we have

$$
\begin{aligned}
& \mathcal{A}_{1, t}^{\varepsilon}=r x \frac{\partial}{\partial x}+w(t) x \frac{\partial}{\partial y}+\frac{1}{2}(\sigma+\varepsilon \hat{\sigma}(t)) x^{2} \frac{\partial^{2}}{\partial x^{2}} \\
& \mathcal{A}_{2, t}^{\varepsilon}=r x \frac{\partial}{\partial x}+\frac{1}{2}(\sigma+\varepsilon \hat{\sigma}(t)) x^{2} \frac{\partial^{2}}{\partial x^{2}}
\end{aligned}
$$

see e.g. Peskir (1998) for the last identity. Note that when specifying the partial differential equations we have for simplicity been rather sloppy in specifying the domains. For the Asian options $(x, y) \in \mathbb{R}_{+}^{2}$, while for the lookback options $(x, y) \in\left\{(a, b) \in \mathbb{R}_{+}^{2} \mid a<b\right\}$. Now, considering the difference $u_{i}(\cdot):=\left(\pi_{i}^{\varepsilon}-\pi_{i}^{0}\right)(\cdot)$, we have

$$
\begin{aligned}
-\frac{\partial u_{i}}{\partial t}(t, x, y)+r u_{i}(t, x, y) & =\mathcal{A}_{i, t}^{0} u_{i}(t, x, y)+g_{i}(t, x, y) \\
1\{i=2\} \frac{\partial u_{i}}{\partial y}(t, x, x) & =0 \\
u_{i}(T, x, y) & =0,
\end{aligned}
$$

with $g_{i}(t, x, y)=\frac{1}{2}\left(2 \varepsilon \hat{\sigma}(t)+\varepsilon^{2} \hat{\sigma}(t)^{2}\right) \frac{\partial^{2} \pi_{i}^{\epsilon}}{\partial x^{2}}(t, x, y)$. According to Karatzas and Shreve (1988) page 369 , the solution is given by

$$
\left(\pi_{i}^{\varepsilon}-\pi_{i}^{0}\right)(t, x, y)=u_{i}(t, x, y)=\int_{t}^{T} \int_{\mathbb{R}_{+}^{2}} G(t, x, y ; \tau, \xi, \varphi) g_{i}(\tau, \xi, \varphi) d \xi d \varphi d \tau
$$


where $G(\cdot)$ is a density function, i.e. non-negative, and called the fundamental solution or the Green's function. Consequently, a sufficient condition for $\left(\pi_{i}^{\varepsilon}-\pi_{i}^{0}\right)(\cdot) \geq 0$ is that $g_{i}(\cdot) \geq 0$ or rather that $\frac{\partial^{2} \pi_{i}^{\varepsilon}}{\partial x^{2}}(\cdot) \geq 0$. Note that we only consider the case where $\hat{\sigma}(\cdot) \geq 0$. Now, using the notation $J^{\varepsilon}(u):=S^{\varepsilon}(u) / S^{\varepsilon}(t)$, we get that

$$
\begin{aligned}
& \pi_{1}^{\varepsilon}(t, x, y)=e^{-r(T-t)} E_{Q}\left[\Phi\left(y+x \int_{t}^{T} w(u) J^{\varepsilon}(u) d u\right) \mid S^{\varepsilon}(t)=x, \int_{0}^{t} w(u) S^{\varepsilon}(u) d u=y\right] \\
& \pi_{2}^{\varepsilon}(t, x, y)=e^{-r(T-t)} E_{Q}\left[\Phi\left(y \vee x \sup _{t \leq u \leq T} J^{\varepsilon}(u)\right) \mid S^{\varepsilon}(t)=x, \sup _{0 \leq u \leq t} S^{\varepsilon}(u)=y\right] .
\end{aligned}
$$

Explicit calculations now yield

$$
\begin{gathered}
\frac{\partial^{2} \pi_{1}^{\varepsilon}}{\partial x^{2}}(t, x, y)=e^{-r(T-t)} E_{Q}\left[\Phi^{\prime \prime}\left(y+x \int_{t}^{T} w(u) J^{\varepsilon}(u) d u\right)\left(\int_{t}^{T} w(u) J^{\varepsilon}(u) d u\right)^{2}\right. \\
\left.\quad \mid S^{\varepsilon}(t)=x, \int_{0}^{t} w(u) S^{\varepsilon}(u) d u=y\right] \\
\frac{\partial^{2} \pi_{2}^{\varepsilon}}{\partial x^{2}}(t, x, y)=e^{-r(T-t)} E_{Q}\left[\Phi^{\prime \prime}\left(x \sup _{t \leq u \leq T} J^{\varepsilon}(u)\right)\left(\sup _{t \leq u \leq T} J^{\varepsilon}(u)\right)^{2} \cdot\right. \\
\left.\cdot 1\left\{x \sup _{t \leq u \leq T} J^{\varepsilon}(u) \geq y\right\} \mid S^{\varepsilon}(t)=x, \sup _{0 \leq u \leq t} S^{\varepsilon}(u)=y\right] \\
+e^{-r(T-t)} \frac{y^{2}}{x^{3}} \Phi^{\prime}(y) p\left(\frac{y}{x}\right),
\end{gathered}
$$

where $p(\cdot)$ denotes the density function of the random variable $\sup _{t \leq u \leq T} J^{\varepsilon}(u)$. Note that the density exists according to lemma 3.4. The result $\frac{\partial^{2} \pi_{i}^{\epsilon}}{\partial x^{2}}(\cdot) \geq 0$ then follows since $\Phi(\cdot)$ is assumed, according to definition 3.1, to be a convex function and therefore the second derivative (or more correctly the second derivative measure) is non-negative and the first derivative is increasing.

Remark A.1 If we consider lookback options in the form $\Phi\left(\inf _{0 \leq t \leq T} S^{\varepsilon}(t)\right)$, then by repeating the arguments we find that $\Pi^{\varepsilon}(\cdot) \geq \Pi(\cdot)$ if $\hat{\sigma}(\cdot) \geq 0$ and the first derivative of the payoff function $\Phi^{\prime}(0) \leq 0$.

\section{Appendix B}

In this Appendix we prove the smoothness of the density of the processes associated with the Asian option, and derive an upper bound for this density. Throughout this section we assume A1 and that the positive bounded weight function $w(\cdot)$ is uniformly bounded away from zero in a neighborhood of zero. In the proof we will make use of the space $\mathbb{D}^{\infty}$, see Nualart (1995) for the precise definition. However, we may think of $\mathbb{D}^{\infty}$ as the space containing all random variables $H$, such that $H$ and $\left\|D^{k} H\right\|_{L^{2}\left([0, T]^{k}\right)}$ belong to $L^{p}(\Omega, F, Q)$ for any $p, k \geq 1$. Hence, 
the random variables in $\mathbb{D}^{\infty}$ are smooth in the sense that they and their iterated stochastic derivatives have moments of all orders.

Theorem B.1 Under the above conditions $Y_{1}^{\varepsilon}(T):=\int_{0}^{T} w(s) S^{\varepsilon}(s) d s \in \mathbb{D}^{\infty}$. The Malliavin covariance

$$
\left[\int_{0}^{T}\left(D_{t} Y_{1}^{\varepsilon}(T)\right)^{2} d t\right]^{-1} \in \cap_{p>1} L^{p}(\Omega, \mathcal{F}, Q),
$$

uniformly in $\varepsilon$. Therefore the density of $Y_{1}^{\varepsilon}(T)$ exists and is infinitely differentiable. Furthermore, this density satisfies for all integers $k \geq 0$ and $y \geq y_{0}>0$ the inequality

$$
\left|\frac{\partial^{k}}{\partial y^{k}} p_{\varepsilon}(y)\right| \leq C \exp \left(-C \ln (y)^{2}\right)
$$

where the constants $C$ and $y_{0}$ are independent of $\varepsilon$.

Proof. We will do the proof under A1 and the assumption that $w(t) \geq w_{0}>0$ in a neighborhood of 0 . First, let us recall that $S^{\varepsilon}(\cdot)$ is defined as the solution to the stochastic differential equation

$$
\left\{\begin{array}{l}
d S^{\varepsilon}(t)=r(t) S^{\varepsilon}(t) d t+\sigma_{\varepsilon}\left(t, S^{\varepsilon}(t)\right) d V(t) \\
S^{\varepsilon}(0)=S_{0}
\end{array} .\right.
$$

According to theorem 2.2.2 in Nualart (1995), the solution $S^{\varepsilon}(t) \in \mathbb{D}^{\infty}$ for all $t \in\left[0, T^{*}\right]$. Therefore, the Malliavin derivatives of any order exist, and are well defined by

$$
D_{u} S^{\varepsilon}(t)=\int_{u}^{t} r(s) D_{u} S^{\varepsilon}(s) d s+\sigma_{\varepsilon}\left(u, S^{\varepsilon}(u)\right)+\int_{u}^{t} \sigma_{\varepsilon}^{\prime}\left(s, S^{\varepsilon}(s)\right) D_{u} S^{\varepsilon}(s) d V(s),
$$

see theorem 2.2.1 in Nualart (1995). This stochastic differential equation is linear in $D_{u} S^{\varepsilon}(t)$, hence the solution is given by

$$
\begin{aligned}
D_{u} S^{\varepsilon}(t) & =\sigma_{\varepsilon}\left(u, S^{\varepsilon}(u)\right) \exp \left(\int_{u}^{t}\left(r(s)-\frac{1}{2} \sigma_{\varepsilon}^{\prime}\left(s, S^{\varepsilon}(s)\right)^{2}\right) d s+\int_{u}^{t} \sigma_{\varepsilon}^{\prime}\left(s, S^{\varepsilon}(s)\right) d V(s)\right) \\
& =\sigma_{\varepsilon}\left(u, S^{\varepsilon}(u)\right) \mathcal{E}^{\varepsilon}(t) \mathcal{E}^{\varepsilon}(u)^{-1},
\end{aligned}
$$

where we have used the same notation as in lemma 3. Since the positive weight function $w(\cdot)$ is bounded and $D_{u} Y_{1}^{\varepsilon}(T)=\int_{u}^{T} w(s) D_{u} S^{\varepsilon}(s) d s$, we immediately see that $Y_{1}^{\varepsilon}(T)$ too is a smooth random variable in $\mathbb{D}^{\infty}$.

Now, let $\alpha>0$ denote the biggest value such that for all $\varepsilon$ the following conditions are satisfied

$$
\sigma_{\varepsilon}(u, x) \geq \sigma_{\min }>0 \quad ; \quad w(u) \geq w_{0}>0
$$


for all $u \leq \alpha$ and $\left|x-S_{0}\right| \leq \alpha$. Hence for $u \leq \tau_{\varepsilon}:=\inf \left\{u \leq \alpha ;\left|S^{\varepsilon}(u)-S_{0}\right| \geq \frac{\alpha}{2}\right\}$, the Malliavin derivative is bounded from below:

$$
\begin{aligned}
D_{u} Y_{1}^{\varepsilon}(T) & =\sigma_{\varepsilon}\left(u, S^{\varepsilon}(u)\right) \mathcal{E}^{\varepsilon}(u)^{-1} \int_{u}^{T} w(s) \mathcal{E}^{\varepsilon}(s) d s \\
& \geq \sigma_{\min } w_{0} \mathcal{E}^{\varepsilon}(u)^{-1} \int_{u}^{\tau_{\varepsilon}} \mathcal{E}^{\varepsilon}(s) d s .
\end{aligned}
$$

Therefore, we get the estimate

$$
\int_{0}^{T}\left(D_{u} Y_{1}^{\varepsilon}(T)\right)^{2} d u \geq \int_{0}^{\tau_{\varepsilon}}\left(D_{u} Y_{1}^{\varepsilon}(T)\right)^{2} d u \geq \sigma_{\min }^{2} w_{0}^{2}\left(\inf _{0 \leq u \leq T} \mathcal{E}^{\varepsilon}(u)\right)^{-2}\left(\inf _{0 \leq u \leq T} \mathcal{E}^{\varepsilon}(u)\right)^{2} \frac{\tau_{\varepsilon}^{3}}{3}
$$

In order to show that the Malliavin covariance $\left[\int_{0}^{T}\left(D_{t} Y_{1}^{\varepsilon}(T)\right)^{2} d t\right]^{-1} \in L^{p}(\Omega, \mathcal{F}, Q)$ for all $p>1$ uniformly in $\varepsilon$, we use the Hölder inequality to conclude that it is sufficient to prove that $\sup _{\varepsilon} E_{Q}\left[\left(\inf _{0 \leq u \leq T} \mathcal{E}^{\varepsilon}(u)\right)^{-p}\right]<+\infty$ and $\sup _{\varepsilon} E_{Q}\left[\tau_{\varepsilon}^{-q}\right]<+\infty$ for any $p \in \mathbb{R}$ and $q>0$.

In order to show that $\sup _{\varepsilon} E_{Q}\left[\left(\inf _{0 \leq u \leq T} \mathcal{E}^{\varepsilon}(u)\right)^{-p}\right]<+\infty$ for any $p \in \mathbb{R}$, note that $r(\cdot)$ is a bounded function and that $\sigma_{\varepsilon}^{\prime}(t, x)$ is uniformly bounded in $t$ and $\varepsilon$. Therefore $\sup _{\varepsilon} E_{Q}\left[\left(\inf _{0 \leq u \leq T} \mathcal{E}^{\varepsilon}(u)\right)^{-p}\right] \leq C \sup _{\varepsilon} E_{Q}\left[\exp \left(c_{1} \sup _{0 \leq u \leq T} \int_{0}^{u} \sigma_{\varepsilon}^{\prime}\left(s, S^{\varepsilon}(s)\right) d V(s)\right)\right]$ for some constants $c_{1}, C$. Since any stochastic integral with bounded quadratic variation is a time changed Brownian motion it is enough to verify that $E_{Q}\left[\exp \left(c_{1} \sup _{0 \leq u \leq c_{2} T} \bar{B}(u)\right)\right]<+\infty$, for any $c_{1} \in \mathbb{R}$ and $c_{2}>0$. This property follows from the reflection principle for the supremum of the $Q$-Brownian motion $\bar{B}(\cdot)$.

Next we show that $\sup _{\varepsilon} E_{Q}\left[\tau_{\varepsilon}^{-q}\right]<+\infty$, for any $q>0$. Note that since $Q\left(\tau_{\varepsilon}<x\right) \leq$ $Q\left(\sup _{0 \leq u \leq x}\left|S^{\varepsilon}(u)-S_{0}\right| \geq \frac{\alpha}{2}\right)$, we can use Chebychev's inequality and then Burkholder's inequality to get:

$$
Q\left(\tau_{\varepsilon}<\theta\right) \leq C_{k} E_{Q}\left[\sup _{0 \leq u \leq \theta}\left|S^{\varepsilon}(u)-S_{0}\right|^{k}\right] \leq C_{k} \theta^{\frac{k}{2}} \quad \text { for any } k \in \mathbb{N} .
$$

Here $C_{k}$ is a constant that does not depend on $\varepsilon$. Finally, since $E_{Q}\left[\tau_{\varepsilon}^{-q}\right]=\int_{0}^{\infty} Q\left(\tau_{\varepsilon}^{-q}>x\right) d x=$ $\int_{0}^{\infty} Q\left(\tau_{\varepsilon} \leq x^{-\frac{1}{q}}\right) d x$ we obtain

$$
\begin{aligned}
E_{Q}\left[\tau_{\varepsilon}^{-q}\right] & =C+\int_{C}^{\infty} Q\left(\tau_{\varepsilon} \leq x^{-\frac{1}{q}}\right) d x \\
& \leq C+\int_{C}^{\infty} C_{k} x^{-\frac{k}{2 q}} d x .
\end{aligned}
$$

The last quantity, which does not depend on $\varepsilon$, is bounded if $k$ is chosen big enough.

Hence, the Malliavin covariance $\left[\int_{0}^{T}\left(D_{t} Y_{1}^{\varepsilon}(T)\right)^{2} d t\right]^{-1} \in L^{p}(\Omega, \mathcal{F}, Q)$ for all $p>1$ uniformly in $\varepsilon$ and therefore, according to corollary 2.1.2 in Nualart (1995), the density of $Y_{1}^{\varepsilon}(T)$ exists and is infinitely differentiable. In order to prove the tail bound for the derivatives of the density 
of $Y_{1}^{\varepsilon}(T)$ we only need to apply Theorem 1.31 in Kusuoka and Stroock (1984). We follow their notation and observe that in our case it is enough to use $\Psi$ as a localization of the random variable $Y_{1}^{\varepsilon}(T)$. That is, $\Psi=\psi\left(Y_{1}^{\varepsilon}(T)\right)$, where $\psi(\cdot)$ is an infinitely differentiable function with bounded derivatives such that its value is 0 in $[0, y]$ and 1 outside of $[0,2 y]$. After a careful analysis of their inequality (1.33) one sees that the proof finishes if one estimates the probability $Q\left(Y_{1}^{\varepsilon}(T)>y\right)$. The estimation of this quantity is done as follows for $y \geq y_{0}>0$. First note that $Q\left(Y_{1}^{\varepsilon}(T)>y\right):=Q\left(\int_{0}^{T} w(s) S^{\varepsilon}(s) d s>y\right) \leq Q\left(\sup _{0 \leq u \leq T} S^{\varepsilon}(u) \geq \frac{1}{T C} y\right)$ for $C=\sup _{0 \leq u \leq T} w(u)$. Therefore there exist constants $c_{i}$ such that:

$$
\begin{aligned}
Q\left(Y_{1}^{\varepsilon}(T)>y\right) & \leq Q\left(\sup _{0 \leq u \leq T} \ln \left(1+S^{\varepsilon}(u)\right)>c_{1}+\ln y\right) \\
& \leq Q\left(\sup _{0 \leq u \leq c_{2} T} \bar{B}(u)>c_{3}+\ln y\right) \\
& \leq c_{4} \exp \left(-c_{4}(\ln y)^{2}\right) .
\end{aligned}
$$

The second inequality above follows from the Itô formula applied to $\ln \left(1+S^{\varepsilon}(u)\right)$ :

$$
d \ln \left(1+S^{\varepsilon}(u)\right)=r(u) \frac{S^{\varepsilon}(u)}{1+S^{\varepsilon}(u)} d u-\frac{\sigma_{\varepsilon}\left(u, S^{\varepsilon}(u)\right)^{2}}{2\left(1+S^{\varepsilon}(u)\right)^{2}} d u+\frac{\sigma_{\varepsilon}\left(u, S^{\varepsilon}(u)\right)}{1+S^{\varepsilon}(u)} d V(u) .
$$

Note that the drift term is bounded, i.e. $\sup _{\varepsilon} \sup _{0 \leq t \leq T}\left|\int_{0}^{t} r(u) \frac{S^{\varepsilon}(u)}{1+S^{\varepsilon}(u)}-\frac{\sigma_{\varepsilon}^{\prime}\left(u, S^{\varepsilon}(u)\right)^{2}}{2\left(1+S^{\varepsilon}(u)\right)^{2}} d u\right|<+\infty$, and that the quadratic variation satisfies $\sup _{\varepsilon} \sup _{0 \leq t \leq T} \int_{0}^{t}\left(\frac{\sigma_{\varepsilon}\left(u, S^{\varepsilon}(u)\right)}{1+S^{\varepsilon}(u)}\right)^{2} d u<+\infty$, since the volatility $\sigma_{\varepsilon}(t, \cdot)$ has at most linear growth uniformly in $t$ according to assumption A1. Therefore, we can as before replace the stochastic integral by a time changed $Q$-Brownian motion $\bar{B}(\cdot)$. Finally, by using the well-known distribution of the supremum of a Brownian motion, we obtain the last inequality and the proof concludes. 